\title{
Dynamics of Scalar Field in Polymer-like Representation
}

\author{
Muxin $\mathrm{Han}^{1,2 *}$ and Yongge $\mathrm{Ma}^{1 \dagger}$ \\ 1. Department of Physics, Beijing Normal University, \\ Beijing 100875, CHINA \\ 2. Horace Hearne Jr. Institute for Theoretical Physics, \\ Louisiana State University, \\ Baton Rouge, LA 70803, USA
}

July 30, 2018

\begin{abstract}
In recent twenty years, loop quantum gravity, a background independent approach to unify general relativity and quantum mechanics, has been widely investigated. We consider the quantum dynamics of a real massless scalar field coupled to gravity in this framework. A Hamiltonian operator for the scalar field can be well defined in the coupled diffeomorphism invariant Hilbert space, which is both self-adjoint and positive. On the other hand, the Hamiltonian constraint operator for the scalar field coupled to gravity can be well defined in the coupled kinematical Hilbert space. There are 1-parameter ambiguities due to scalar field in the construction of both operators. The results heighten our confidence that there is no divergence within this background independent and diffeomorphism invariant quantization approach of matter coupled to gravity. Moreover, to avoid possible quantum anomaly, the master constraint programme can be carried out in this coupled system by employing a self-adjoint master constraint operator on the diffeomorphism invariant Hilbert space.
\end{abstract}

Keywords: loop quantum gravity, quantum dynamics, scalar field, background independence.

PACS number(s): 04.60.Pp, 04.60.Ds

\section{Introduction}

The research on quantum gravity theory is rather active. Many quantization programmes for gravity are being carried out (for a summary see e.g. [1]). In these differ-

*Email address: mhan1@1su.edu

†Email address: mayg@bnu.edu.cn 
ent kinds of approaches, the idea of loop quantum gravity is motivated by researchers in the community of general relativity. It follows closely the thoughts of general relativity, and hence it is a quantum theory born with background independency [2]. For recent review in this field, we refer to [3][4][5].

To apply the background independent quantization technique, one first casts general relativity into the Hamiltonian formalism of a diffeomorphism invariant YangMills gauge field theory with a compact internal gauge group [6]|7]. The kinematical Hilbert space $\mathcal{H}_{\text {kin }}^{G R}$ of the quantum theory is then constructed rigorously. One can even solve the Gaussian and diffeomorphism constraints to arrive at a diffeomorphism invariant Hilbert space [8]. Certain geometrical operators are shown to have discrete spectra in the kinematical Hilbert space [9][10][11][12][13]. However, some important elements in this approach are not yet understood. Despite the systematic efforts in constructing the Hamiltonian constraint operator[14][15] and the master constraint operator[16][17][18], the dynamics of the quantum theory has not been fully understood, especially if one wants to include all known matter fields. The primary goal of this paper is to apply the loop quantization technique to a scalar field and check whether the quantum dynamics can be well defined. Since we will use the developed polymer-like kinematical description of the scalar field[19][20], it can be considered as a development of the construction for quantum Higgs fields in Refs. [21] and [22].

In section 2, the Hamiltonian formalism of a massless real scalar field coupled to gravity is obtained in generalized Palatini formulation. For readers' convenience, the loop quantum kinematical setting of a real scalar field coupled to gravity is also introduced. We then show in section 3 that an operator corresponding to the Hamiltonian of the scalar field can be well defined on the coupled diffeomorphism invariant Hilbert space. It is even positive and self-adjoint. Thus quantum gravity acts exactly as a natural regulator for the quantum scalar field in the polymer representation. In section 4, to study the whole dynamical system of the scalar field coupled to gravity, a Hamiltonian constraint operator is defined in the coupled kinematical Hilbert space. Moreover, the contribution of the scalar field to the Hamiltonian constraint can be promoted to a positive self-adjoint operator. Similar to the gravitational Hamiltonian constraint, there is an one-parameter ambiguity in defining both the Hamiltonian operator and the constraint operator due to the scalar field. To avoid possible quantum anomaly and find physical Hilbert space, the programme of master constraint for the coupled system is discussed in section 5. A self-adjoint master operator is obtained in the diffeomorphism invariant Hilbert space, which assures the feasibility of the programme.

\section{Polymer-like representation for scalar field coupled to gravity}

Consider first the classical dynamics of a real massless scale field $\phi$ coupled to gravity on a 4-manifold $M$. The coupled generalized Palatini action reads

$$
S\left[e_{K}^{\beta}, \omega_{\alpha}^{I J}, \phi\right]=S_{p}\left[e_{K}^{\beta}, \omega_{\alpha}^{I J}\right]+S_{K G}\left[e_{K}^{\beta}, \phi\right],
$$


where

$$
\begin{array}{r}
S_{p}\left[e_{K}^{\beta}, \omega_{\alpha}^{I J}\right]=\frac{1}{2 \kappa} \int_{M} d^{4} x(e) e_{I}^{\alpha} e_{J}^{\beta}\left(\Omega_{\alpha \beta}^{I J}+\frac{1}{2 \gamma} \epsilon_{K L}^{I J} \Omega_{\alpha \beta}^{K L}\right), \\
S_{K G}\left[e_{K}^{\beta}, \phi\right]=-\frac{\alpha_{M}}{2} \int_{M} d^{4} x(e) \eta^{I J} e_{I}^{\alpha} e_{J}^{\beta}\left(\partial_{\alpha} \phi\right) \partial_{\beta} \phi
\end{array}
$$

here $e_{K}^{\beta}$ and $\omega_{\alpha}^{I J}$ are respectively the tetrad and Lorentz connection on $M$, the real number $\gamma, k$ and $\alpha_{M}$ are respectively the Barbero-Immirzi parameter, the gravitational constant and the coupling constant. From now on we use $\alpha, \beta, \cdots$ for 4 -dimensional spacetime indices and $I, J, \cdots$ for internal Lorentz indices, $a, b, \cdots$ for 3 -dimensional spacial indices and $i, j, \cdots$ for internal $S U(2)$ indices. After $3+1$ decomposition and Legendre transformation, similar to the case in Palatini formalism[23], we obtain the total Hamiltonian of the coupling system on the 3-manifold $\Sigma$ as:

$$
\mathcal{H}_{\text {tot }}=\int_{\Sigma}\left(\Lambda^{i} G_{i}+N^{a} \mathcal{V}_{a}+N C\right),
$$

where $\Lambda^{i}, N^{a}$ and $N$ are Lagrange multipliers, and the Gaussian, diffeomorphism and Hamiltonian constraints are expressed respectively as:

$$
\begin{aligned}
G_{i} & =D_{a} \widetilde{P}_{i}^{a}:=\partial_{a} \widetilde{P}_{i}^{a}+\epsilon_{i j}{ }^{k} A_{a}^{j} \widetilde{P}_{k}^{a}, \\
\mathcal{V}_{a} & =\widetilde{P}_{i}^{b} F_{a b}^{i}-A_{a}^{i} G_{i}+\widetilde{\pi} \partial_{a} \phi, \\
C & =\frac{\kappa \gamma^{2}}{2 \sqrt{|\operatorname{det} q|}} \widetilde{P}_{i}^{a} \widetilde{P}_{j}^{b}\left[\epsilon^{i j}{ }_{k} F_{a b}^{k}-2\left(1+\gamma^{2}\right) K_{[a}^{i} K_{b]}^{j}\right] \\
& +\frac{1}{\sqrt{|\operatorname{det} q|}}\left[\frac{\kappa^{2} \gamma^{2} \alpha_{M}}{2} \delta^{i j} \widetilde{P}_{i}^{a} \widetilde{P}_{j}^{b}\left(\partial_{a} \phi\right) \partial_{b} \phi+\frac{1}{2 \alpha_{M}} \widetilde{\pi}^{2}\right],
\end{aligned}
$$

here the conjugate pair for gravity consists of the $S U(2)$ connection $A_{a}^{i}$ and the densitized triad $\widetilde{P}_{j}^{b}, F_{a b}^{i}$ is the curvature of $A_{a}^{i}$, and $\widetilde{\pi}$ denotes the momentum conjugate to $\phi$. Thus one has the elementary Poisson brackets

$$
\begin{aligned}
\left\{A_{a}^{i}(x), \widetilde{P}_{j}^{b}(y)\right\} & =\delta_{b}^{a} \delta_{j}^{i} \delta(x-y), \\
\{\phi(x), \widetilde{\pi}(y)\} & =\delta(x-y) .
\end{aligned}
$$

Note that the second term of the Hamiltonian constraint (4) is just the Hamiltonian of the real scalar field.

Now we introduce the background independent quantization of a real scalar field coupled to gravity, following the polymer representation of the scalar field [19][20]. The classical configuration space, $\mathcal{U}$, consists of all real-valued smooth functions $\phi$ on $\Sigma$. Given a set of finite number of points $X=\left\{x_{1}, \ldots, x_{N}\right\}$ in $\Sigma$, denote $C y l_{X}$ the vector space generated by finite linear combinations of the following functions of $\phi$ :

$$
\Pi_{X, \lambda}(\phi):=\prod_{x_{j} \in X} \exp \left[i \lambda_{j} \phi\left(x_{j}\right)\right],
$$


where $\lambda \equiv\left(\lambda_{1}, \lambda_{2}, \cdots, \lambda_{N}\right)$ are arbitrary real numbers. It is obvious that $C y l_{X}$ has the structure of a $*$-algebra. The space $C y l$ of all cylindrical functions on $\mathcal{U}$ is defined by

$$
C y l:=\cup_{X} C y l_{X} .
$$

Completing $C y l$ with respect to the sup norm, one obtains a unital Abelian $C^{*}$-algebra $\overline{C y l}$. Thus one can use the GNS structure to construct its cyclic representations. A preferred positive linear functional $\omega_{0}$ on $\overline{C y l}$ is defined by

$$
\omega_{0}\left(\Pi_{X, \lambda}\right)= \begin{cases}1 & \text { if } \lambda_{j}=0 \forall j \\ 0 & \text { otherwise }\end{cases}
$$

which defines a diffeomorphism-invariant faithful Borel measure $\mu$ on $\mathcal{U}$ as

$$
\int_{\mathcal{U}} d \mu\left(\Pi_{X, \lambda}\right)= \begin{cases}1 & \text { if } \lambda_{j}=0 \forall j \\ 0 & \text { otherwise }\end{cases}
$$

Thus one obtains the Hilbert space, $\mathcal{H}_{k i n}^{K G} \equiv L^{2}(\overline{\mathcal{U}}, d \mu)$, of square integrable functions on a compact topological space $\overline{\mathcal{U}}$ with respect to $\mu$, where $\overline{C y l}$ acts by multiplication. The quantum configuration space $\overline{\mathcal{U}}$ is called the Gel'fand spectrum of $\overline{C y l}$. More concretely, for a single point set $X_{0} \equiv\left\{x_{0}\right\}, C y l_{X_{0}}$ is the space of all almost periodic functions on a real line $\mathbf{R}$. The Gel'fand spectrum of the corresponding $C^{*}$-algebra $\overline{C y l}_{X_{0}}$ is the Bohr completion $\overline{\mathbf{R}}_{x_{0}}$ of $\mathbf{R}$ [19], which is a compact topological space such that $\overline{C y l}_{X_{0}}$ is the $C^{*}$-algebra of all continuous functions on $\overline{\mathbf{R}}_{x_{0}}$. Since $\mathbf{R}$ is densely embedded in $\overline{\mathbf{R}}_{x_{0}}, \overline{\mathbf{R}}_{x_{0}}$ can be regarded as a completion of $\mathbf{R}$.

Given a pair $\left(x_{0}, \lambda_{0}\right)$, there is an elementary configuration for the scalar field, the so-called point holonomy,

$$
U\left(x_{0}, \lambda_{0}\right):=\exp \left[i \lambda_{0} \phi\left(x_{0}\right)\right] .
$$

It corresponds to a configuration operator $\hat{U}\left(x_{0}, \lambda_{0}\right)$, which acts on any cylindrical function $\psi(\phi) \in \mathcal{H}_{\text {kin }}^{K G}$ by

$$
\hat{U}\left(x_{0}, \lambda_{0}\right) \psi(\phi)=U\left(x_{0}, \lambda_{0}\right) \psi(\phi) .
$$

All these operators are unitary. But since the family of operators $\hat{U}\left(x_{0}, \lambda\right)$ fails to be weakly continuous in $\lambda$, there is no operator $\hat{\phi}(x)$ on $\mathcal{H}_{\text {kin }}^{K G}$. The momentum functional smeared on a 3-dimensional region $R \subset \Sigma$ is expressed by

$$
\pi(R):=\int_{R} d^{3} x \widetilde{\pi}(x) .
$$

The Poisson bracket between the momentum functional and a point holonomy can be easily calculated to be

$$
\{\pi(R), U(x, \lambda)\}=-i \lambda \chi_{R}(x) U(x, \lambda),
$$

where $\chi_{R}(x)$ is the characteristic function for the region $R$. So the momentum operator is defined by the action on scalar network functions $\Pi_{c=(X, \lambda)}$ as

$$
\hat{\pi}(R) \Pi_{c}(\phi):=i \hbar\left\{\pi(R), \Pi_{c}(\phi)\right\}=\hbar\left[\sum_{x_{j} \in X} \lambda_{j} \chi_{R}\left(x_{j}\right)\right] \Pi_{c}(\phi) .
$$


It is clear from Eq. (6) that an orthonormal basis in $\mathcal{H}_{k i n}^{K G}$ is given by the so-called scalar network functions $\Pi_{c}(\phi)$, where $c$ denotes $(X(c), \lambda)$ and $\lambda \equiv\left(\lambda_{1}, \lambda_{2}, \cdots, \lambda_{N}\right)$ are nonzero real numbers now. So the total kinematical Hilbert space $\mathcal{H}_{\text {kin }}$ is the direct product of the kinematical Hilbert space $\mathcal{H}_{\text {kin }}^{G R}$ for gravity and the kinematical Hilbert space for real scalar field, i.e., $\mathcal{H}_{\text {kin }}:=\mathcal{H}_{\text {kin }}^{G R} \otimes \mathcal{H}_{\text {kin }}^{K G}$. Let $\Pi_{s}(A)$ be the spin network basis in $\mathcal{H}_{\text {kin }}^{G R}$ labeled by $s[11][24]$. Then the state $\Pi_{s, c} \equiv \Pi_{s}(A) \otimes \Pi_{c}(\phi) \in C y l_{\gamma(s)}(\overline{\mathcal{A}}) \otimes$ $C y l_{X(c)} \equiv C y l_{\gamma(s, c)}(\overline{\mathcal{A}}) \otimes C y l_{\gamma(s, c)}(\overline{\mathcal{U}})$ is a gravity-scalar cylindrical function on graph $\gamma(s, c) \equiv \gamma(s) \cup X(c)$. Note that generally $X(c)$ may not coincide with the vertices of the graph $\gamma(s)$. It is straightforward to see that all of these functions constitutes a complete set of orthonormal basis in $\mathcal{H}_{\text {kin }}$ as

$$
<\Pi_{s^{\prime}, c^{\prime}}(A, \phi) \mid \Pi_{s, c}(A, \phi)>_{k i n}=\delta_{s^{\prime} s} \delta_{c^{\prime} c} .
$$

Note that none of $\mathcal{H}_{\text {kin }}, \mathcal{H}_{\text {kin }}^{G R}$ and $\mathcal{H}_{\text {kin }}^{K G}$ is a separable Hilbert space.

Now we can consider the quantum dynamics and impose the quantum constraints on $\mathcal{H}_{\text {kin }}$. Firstly, the Gaussian constraint can be solved independently of $\mathcal{H}_{\text {kin }}^{K G}$, since it only involves gravitational field. It is also expected that the diffeomorphism constraint can be implemented by the group averaging strategy in the similar way as that in the case of pure gravity. Given a spatial diffeomorphism transformation $\varphi$, a unitary transformation $\hat{U}_{\varphi}$ is induced by $\varphi$ in the Hilbert space $\mathcal{H}_{\text {kin }}$, which is expressed as

$$
\hat{U}_{\varphi} \Pi_{s=(\gamma(s), \mathbf{j}, \mathbf{N}), c=(X(c), \lambda)}=\Pi_{\varphi \circ s=(\varphi(\gamma(s)), \mathbf{j}, \mathbf{N}), \varphi \circ c=(\varphi(X(c)), \lambda)} .
$$

Then the differomorphism invariant spin-scalar-network functions are defined by group averaging as

$$
\Pi_{[s, c]}:=\frac{1}{n_{\gamma(s, c)}} \sum_{\varphi \in D_{i f f / D i f f} f_{\gamma(s, c)}} \sum_{\varphi^{\prime} \in G S_{\gamma(s, c)}} \hat{U}_{\varphi} \hat{U}_{\varphi^{\prime}} \Pi_{s, c},
$$

where $\operatorname{Diff} f_{\gamma}$ is the set of diffeomorphisms leaving the colored graph $\gamma$ invariant, $G S_{\gamma}$ denotes the graph symmetry quotient group Diff $f_{\gamma} / T$ Diff $f_{\gamma}$ where $T$ Diff $f_{\gamma}$ is the set of the diffeomorphisms which is trivial on the graph $\gamma$, and $n_{\gamma}$ is the number of elements in $G S_{\gamma}$. Following the standard strategy in quantization of pure gravity, an inner product can be defined on the vector space spanned by the diffeomorphism invariant spin-scalarnetwork functions such that they form an orthonormal basis as:

$$
<\Pi_{[s, c]} \mid \Pi_{\left[s^{\prime}, c^{\prime}\right]}>_{D i f f}:=\Pi_{[s, c]}\left[\Pi_{s^{\prime}, c^{\prime} \in\left[s^{\prime}, c^{\prime}\right]}\right]=\delta_{[s, c],\left[s^{\prime}, c^{\prime}\right]}
$$

After the completion procedure, we obtain the expected Hilbert space of diffeomorphism invariant states for the scalar field coupled to gravity, which is denoted by $\mathcal{H}_{\text {Diff }}$. In the following sections, we would like to discuss the quantum dynamical properties of the polymer-like scalar field coupled to gravity.

\section{Diffeormorphism invariant quantum Hamiltonian of scalar field}

In the present section, we first consider the quantum scalar field on a fluctuating background. A similar idea was considered in Ref.[25], where a Hamiltonian operator with 
respect to a $U(1)$ group representation of scalar field is defined on a kinematical Hilbert space $\mathcal{H}_{\text {kin }}$ of matter coupled to gravity. Then an effective Hamiltonian operator of the scalar field can be constructed as a quadratic form via

$$
\begin{aligned}
& <\psi_{\text {matter }}, \hat{H}_{\text {matter }}^{\text {eff }}(m) \psi_{\text {matter }}^{\prime}>_{\text {kin }}^{K G} \\
:=\quad & <\psi_{\text {grav }}(m) \otimes \psi_{\text {matter }}, \hat{H}_{\text {matter }} \psi_{\text {grav }}(m) \otimes \psi_{\text {matter }}^{\prime}>_{\text {kin }^{\prime}},
\end{aligned}
$$

where $\psi_{\text {grav }}(m) \in \mathcal{H}_{\text {kin }}^{G R}$ presents a semiclassical state of gravity approximating some classical spacetime background $m$ where the quantum scalar field lives. Thus the effective Hamiltonian operator $\hat{H}_{\text {matter }}^{\text {eff }}(m)$ of scalar field contains also the information of the fluctuating background metric. In the light of this idea, we will construct a Hamiltonian operator $\hat{\mathcal{H}}_{K G}$ for scalar field in the polymer-like representation. It turns out that the Hamiltonian operator can be defined in the Hilbert space $\mathcal{H}_{\text {Diff }}$ of diffeomorphism invariant states for scalar field coupled to gravity without UV-divergence. So the quantum dynamics of the scalar field is obtained in a diffeomorphism invariant way, which is expected in the programme of loop quantum gravity. Thus, here an effective Hamiltonian operator of the scalar field could be extracted in $\mathcal{H}_{\text {Diff }}$ by defining $<\Psi_{[m]}(A, \phi), \hat{\mathcal{H}}_{K G} \Psi_{[m]}(A, \phi)>_{\text {Diff }}$ to be its expectation value on diffeomorphism invariant states $\Psi(\phi)$ of scalar field, where the diffeomorphism invariant semiclassical state $\Psi_{[m]}(A)$ represents certain fluctuating geometry with spatial diffeomorphism invariance, and the label $[m]$ denotes the classical geometry approximated by $\Psi_{[m]}(A)$. Moreover, the quadratic properties of the scalar field Hamiltonian will provide powerful functional analytic tools in the quantization procedure, such that the self-adjointness of the Hamiltonian operator can be proved by the theorem in functional analysis.

\subsection{Regularization of the Hamiltonian}

In a suitable gauge, the classical Hamiltonian for the massless scalar field on the 3manifold $\Sigma$ can be given by

$$
\begin{aligned}
\mathcal{H}_{K G} & =\mathcal{H}_{K G, \phi}+\mathcal{H}_{K G, K i n} \\
& =\int_{\Sigma} d^{3} x\left[\frac{\kappa^{2} \gamma^{2} \alpha_{M}}{2} \frac{1}{\sqrt{|\operatorname{det} q|}} \delta^{i j} \widetilde{P}_{i}^{a} \widetilde{P}_{j}^{b}\left(\partial_{a} \phi\right) \partial_{b} \phi+\frac{1}{2 \alpha_{M}} \frac{1}{\sqrt{|\operatorname{det} q|}} \widetilde{\pi}^{2}\right] .
\end{aligned}
$$

We will employ the following identities:

$$
\widetilde{P}_{i}^{a}=\frac{1}{2 \kappa \gamma} \widetilde{\eta}^{a b c} \epsilon_{i j k} e_{b}^{j} e_{c}^{k} \quad \text { and } \quad e_{a}^{i}(x)=\frac{2}{\kappa \gamma}\left\{A_{a}^{i}(x), V_{U_{x}}\right\},
$$

where $\widetilde{\eta}^{a b c}$ denotes the Levi-Civita tensor tensity and $V_{U_{x}}$ is the volume of an arbitrary neighborhood $U_{x}$ containing the point $x$. By using the point-splitting strategy, the regulated version of the Hamiltonian (11) is obtained as:

$$
\begin{aligned}
\mathcal{H}_{K G, \phi}= & \frac{\kappa^{2} \gamma^{2} \alpha_{M}}{2} \int_{\Sigma} d^{3} y \int_{\Sigma} d^{3} x \chi_{\epsilon}(x-y) \delta^{i j} \times \\
& \frac{1}{\sqrt{V_{U_{x}^{\epsilon}}}} \widetilde{P}_{i}^{a}(x)\left(\partial_{a} \phi(x)\right) \frac{1}{\sqrt{V_{U_{y}^{\epsilon}}}} \widetilde{P}_{j}^{b}(y) \partial_{b} \phi(y)
\end{aligned}
$$




$$
\begin{aligned}
= & \frac{32 \alpha_{M}}{\kappa^{4} \gamma^{4}} \int_{\Sigma} d^{3} y \int_{\Sigma} d^{3} x \chi_{\epsilon}(x-y) \delta^{i j} \times \\
& \widetilde{\eta}^{a e c}\left(\partial_{a} \phi(x)\right) \operatorname{Tr}\left(\tau_{i}\left\{\mathbf{A}_{e}(x), V_{U_{x}^{\epsilon}}^{3 / 4}\right\}\left\{\mathbf{A}_{c}(x), V_{U_{x}^{\epsilon}}^{3 / 4}\right\}\right) \times \\
& \widetilde{\eta}^{b f d}\left(\partial_{b} \phi(y)\right) \operatorname{Tr}\left(\tau_{j}\left\{\mathbf{A}_{f}(y), V_{U_{y}^{\epsilon}}^{3 / 4}\right\}\left\{\mathbf{A}_{d}(y), V_{U_{y}^{\epsilon}}^{3 / 4}\right\}\right), \\
\mathcal{H}_{K G, \text { in }}= & \frac{1}{2 \alpha_{M}} \int_{\Sigma} d^{3} x \widetilde{\pi}(x) \int_{\Sigma} d^{3} x \widetilde{\pi}(y) \times \\
& \int_{\Sigma} d^{3} u \frac{\operatorname{det}\left(e_{a}^{i}(u)\right)}{\left(V_{U_{u}^{\epsilon}}\right)^{3 / 2}} \int_{\Sigma} d^{3} w \frac{\operatorname{det}\left(e_{a}^{i}(v)\right)}{\left(V_{U_{w}^{\epsilon}}\right)^{3 / 2}} \chi_{\epsilon}(x-y) \chi_{\epsilon}(u-x) \chi_{\epsilon}(w-y) \\
= & \frac{1}{2 \alpha_{M}} \frac{2^{8}}{9(\kappa \gamma)^{6}} \int_{\Sigma} d^{3} x \widetilde{\pi}(x) \int_{\Sigma} d^{3} x \widetilde{\pi}(y) \times \\
& \int_{\Sigma} d^{3} u \widetilde{\eta}^{a b c} \operatorname{Tr}\left(\left\{\mathbf{A}_{a}(u), \sqrt{V_{U_{u}^{\epsilon}}}\right\}\left\{\mathbf{A}_{b}(u), \sqrt{V_{U_{u}^{\epsilon}}}\right\}\left\{\mathbf{A}_{c}(u), \sqrt{V_{U_{u}^{\epsilon}}}\right\}\right) \times \\
& \int_{\Sigma} d^{3} w \widetilde{\eta}^{d e f} \operatorname{Tr}\left(\left\{\mathbf{A}_{d}(w), \sqrt{V_{U_{w}^{\epsilon}}}\right\}\left\{\mathbf{A}_{e}(w), \sqrt{V_{U_{w}^{\epsilon}}}\right\}\left\{\mathbf{A}_{f}(w), \sqrt{V_{U_{w}^{\epsilon}}}\right\}\right) \times \\
& \chi_{\epsilon}(x-y) \chi_{\epsilon}(u-x) \chi_{\epsilon}(w-y),
\end{aligned}
$$

where we denote $\mathbf{A}_{a} \equiv A_{a}^{i} \tau_{i}, \chi_{\epsilon}(x-y)$ is the characteristic function of a box containing $x$ with scale $\epsilon$ such that $\lim _{\epsilon \rightarrow 0} \chi_{\epsilon}(x-y) / \epsilon^{3}=\delta(x-y)$, and $V_{U_{x}^{\epsilon}}$ is the volume of the box. In order to quantize the Hamiltonian as a well-defined operator in polymer-like representation, we have to express the classical formula of $\mathcal{H}_{K G}$ in terms of elementary variables with clear quantum analogs. This can be realized by introducing a triangulation $T(\epsilon)$ of $\Sigma$, where the parameter $\epsilon$ describes how fine the triangulation is. The quantity regulated on the triangulation is required to have correct limit when $\epsilon \rightarrow 0$. Given a tetrahedron $\Delta \in T(\epsilon)$, we use $\left\{s_{i}(\Delta)\right\}_{i=1,2,3}$ to denote the three outgoing oriented segments in $\Delta$ with a common beginning point $v(\Delta)=s\left(s_{i}(\Delta)\right)$ and use $a_{i j}(\Delta)$ to denote the arcs connecting the end points of $s_{i}(\Delta)$ and $s_{j}(\Delta)$. Then several loops $\alpha_{i j}(\Delta)$ are formed by $\alpha_{i j}(\Delta):=s_{i}(\Delta) \circ a_{i j}(\Delta) \circ s_{j}(\Delta)^{-1}$. Thus we have the identities:

$$
\left\{\int_{s(\Delta)} d t \mathbf{A}_{a} \dot{s}^{a}(t), V_{U_{s(s(\Delta))}^{\epsilon}}^{3 / 4}\right\}=-A(s(\Delta))^{-1}\left\{A(s(\Delta)), V_{U_{s(s(\Delta))}^{3 / 4}}^{3 / 4}\right\}+o(\epsilon),
$$

and

$$
\int_{s(\Delta)} d t \partial_{a} \phi \dot{s}^{a}(t)=\frac{1}{i \lambda} U(s(s(\Delta)), \lambda)^{-1}[U(t(s(\Delta)), \lambda)-U(s(s(\Delta)), \lambda)]+o(\epsilon)
$$

for nonzero $\lambda$, where $s(s(\Delta))$ and $t(s(\Delta))$ denote respectively the beginning and end points of segment $s(\Delta)$ with scale $\epsilon$ associated with a tetrahedron $\Delta$. Regulated on the triangulation, the classical Hamiltonian of scalar field reads

$$
\begin{aligned}
\mathcal{H}_{K G, \phi}^{\epsilon}= & -\frac{4 \alpha_{M}}{9 \kappa^{4} \gamma^{4}} \sum_{\Delta^{\prime} \in T(\epsilon)} \sum_{\Delta \in T(\epsilon)} \chi_{\epsilon}\left(v(\Delta)-v\left(\Delta^{\prime}\right)\right) \delta^{i j} \times \\
& \epsilon^{l m n} \frac{1}{\lambda} U(v(\Delta), \lambda)^{-1}\left[U\left(t\left(s_{l}(\Delta)\right), \lambda\right)-U(v(\Delta), \lambda)\right] \times \\
& \operatorname{Tr}\left(\tau_{i} A\left(s_{m}(\Delta)\right)^{-1}\left\{A\left(s_{m}(\Delta)\right), V_{U_{v(\Delta)}^{\epsilon}}^{3 / 4}\right\} A\left(s_{n}(\Delta)\right)^{-1}\left\{A\left(s_{n}(\Delta)\right), V_{U_{v(\Delta)}^{\epsilon}}^{3 / 4}\right\}\right) \times
\end{aligned}
$$




$$
\begin{aligned}
& \epsilon^{k p q} \frac{1}{\lambda} U\left(v\left(\Delta^{\prime}\right), \lambda\right)^{-1}\left[U\left(t\left(s_{k}\left(\Delta^{\prime}\right)\right), \lambda\right)-U\left(v\left(\Delta^{\prime}\right), \lambda\right)\right] \times \\
& \operatorname{Tr}\left(\tau_{j} A\left(s_{p}\left(\Delta^{\prime}\right)\right)^{-1}\left\{A\left(s_{p}\left(\Delta^{\prime}\right)\right), V_{U_{v\left(\Delta^{\prime}\right)}^{\epsilon}}^{3 / 4}\right\} A\left(s_{q}\left(\Delta^{\prime}\right)\right)^{-1}\left\{A\left(s_{q}\left(\Delta^{\prime}\right)\right), V_{U_{v\left(\Delta^{\prime}\right)}^{\epsilon}}^{3 / 4}\right\}\right), \\
& \mathcal{H}_{\text {KG,Kin }}^{\epsilon}=\frac{16}{81 \alpha_{M}(\kappa \gamma)^{6}} \sum_{\Delta \in T(\epsilon)} \sum_{\Delta^{\prime} \in T(\epsilon)} \pi(\Delta) \pi\left(\Delta^{\prime}\right) \times \\
& \sum_{\Delta^{\prime \prime} \in T(\epsilon)} \epsilon^{i m n} \operatorname{Tr}\left(A\left(s_{i}\left(\Delta^{\prime \prime}\right)\right)^{-1}\left\{A\left(s_{i}\left(\Delta^{\prime \prime}\right)\right), \sqrt{V_{U_{v\left(\Delta^{\prime \prime}\right)}^{\epsilon}}}\right\} \times\right. \\
& A\left(s_{m}\left(\Delta^{\prime \prime}\right)\right)^{-1}\left\{A\left(s_{m}\left(\Delta^{\prime \prime}\right)\right), \sqrt{V_{U_{v\left(\Delta^{\prime \prime}\right)}^{\epsilon}}}\right\} \times \\
& \left.A\left(s_{n}\left(\Delta^{\prime \prime}\right)\right)^{-1}\left\{A\left(s_{n}\left(\Delta^{\prime \prime}\right)\right), \sqrt{V_{U_{v\left(\Delta^{\prime \prime}\right)}^{\epsilon}}}\right\}\right) \times \\
& \sum_{\Delta^{\prime \prime \prime} \in T(\epsilon)} \epsilon^{j k l} \operatorname{Tr}\left(A\left(s_{j}\left(\Delta^{\prime \prime \prime}\right)\right)^{-1}\left\{A\left(s_{j}\left(\Delta^{\prime \prime \prime}\right)\right), \sqrt{V_{U_{v\left(\Delta^{\prime \prime \prime}\right)}^{\epsilon}}}\right\} \times\right. \\
& A\left(s_{k}\left(\Delta^{\prime \prime \prime}\right)\right)^{-1}\left\{A\left(s_{k}\left(\Delta^{\prime \prime \prime}\right)\right), \sqrt{V_{U_{v\left(\Delta^{\prime \prime \prime}\right)}^{\epsilon}}}\right\} \times \\
& A\left(s_{l}\left(\Delta^{\prime \prime \prime}\right)\right)^{-1}\left\{A\left(s_{l}\left(\Delta^{\prime \prime \prime}\right)\right), \sqrt{\left.V_{U_{v\left(\Delta^{\prime \prime \prime}\right.}^{\epsilon}}\right\}}\right) \times \\
& \chi_{\epsilon}\left(v(\Delta)-v\left(\Delta^{\prime}\right)\right) \chi_{\epsilon}\left(v\left(\Delta^{\prime \prime}\right)-v(\Delta)\right) \chi_{\epsilon}\left(v\left(\Delta^{\prime \prime \prime}\right)-v\left(\Delta^{\prime}\right)\right),
\end{aligned}
$$

where the overall numerical factors are got from the scale of a tetrahedron to an octahedron. Note that the above regularization method is in complete analogy with that used for Higgs field in Ref.[22]. However, our regularization formula of scalar field Hamiltonian is explicitly dependent on the parameter $\lambda$, which will leads to a kind of quantization ambiguity of the real scalar field dynamics in polymer-like representation.

\subsection{Quantization of the Hamiltonian}

Since all constituents in the expression (12) have clear quantum analogs, one can quantize it as an operator by replacing the constituents by the corresponding operators and Poisson brackets by canonical commutators. Then the regulator should be removed by $\epsilon \rightarrow 0$ with respect to a suitable operator topology. Now we begin to construct the Hamiltonian operator. Given a spin-scalar-network function $\Pi_{s, c}$, in order to ensure that the final operator is diffeomorphism covariant, and cylindrically consistent (up to diffeomorphisms), one can make the triangulation $T(\epsilon)$ adapted to the graph $\gamma(s, c)$ of $\Pi_{s, c}$ according to the strategy developed in Ref.[14] with the following properties.

- The graph $\gamma(s, c)$ is embedded in $T(\epsilon)$ for all $\epsilon$, so that every vertex $v$ of $\gamma(s, c)$ coincides with a vertex $v(\Delta)$ in $T(\epsilon)$.

- For every triple of edges $\left(e_{1}, e_{2}, e_{3}\right)$ of $\gamma(s, c)$ such that $v=s\left(e_{1}\right)=s\left(e_{2}\right)=s\left(e_{3}\right)$, there is a tetrahedra $\Delta \in T(\epsilon)$ such that $v=v(\Delta)$ and $s_{i}(\Delta) \subset e_{i}, \forall i=1,2,3$. We denote such a tetrahedra as $\Delta_{s_{1}, s_{2}, s_{3}}^{0}$.

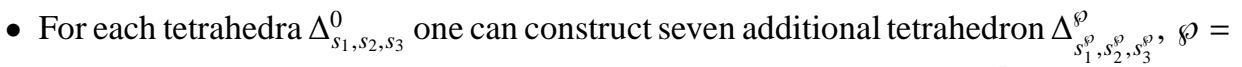
$1, \ldots, 7$, by backward analytic extensions of $s_{i}(\Delta)$ so that $U_{s_{1}, s_{2}, s_{3}}:=\cup_{\wp=0}^{7} \Delta_{s_{1}^{\vartheta}, s_{2}^{\vartheta}, s_{3}^{\vartheta}}^{\wp}$ is a neighborhood of $v$. 
- The triangulation must be fine enough so that the neighborhoods $U(v):=\cup_{s_{1}, s_{2}, s_{3}} U_{s_{1}, s_{2}, s_{3}}(v)$ are disjoint for different vertices $v$ and $v^{\prime}$ of $\gamma(s, c)$. Thus for any open neighborhood $U_{\gamma(s, c)}$ of the graph $\gamma(s, c)$, there exists a triangulation $T(\epsilon)$ such that $\cup_{v \in V(\gamma(s, c))} U(v) \subseteq U_{\gamma(s, c)}$.

- The distance between a vertex $v(\Delta)$ and the corresponding $\operatorname{arcs} a_{i j}(\Delta)$ is described by the parameter $\epsilon$. For any two different $\epsilon$ and $\epsilon^{\prime}$, the $\operatorname{arcs} a_{i j}\left(\Delta^{\epsilon}\right)$ and $a_{i j}\left(\Delta^{\epsilon^{\prime}}\right)$ with respect to one vertex $v(\Delta)$ are analytically diffeomorphic with each other.

- With the triangulation $T(\epsilon)$, the integral over $\Sigma$ is replaced by the Riemanian sum:

$$
\begin{aligned}
\int_{\Sigma} & =\int_{U_{\gamma(s, c)}}+\int_{\Sigma-U_{\gamma(s, c)}}, \\
\int_{U_{\gamma(s, c)}} & =\sum_{v \in V(\gamma(s, c))} \int_{U(v)}+\int_{U_{\gamma(s, c)}-\cup_{v} U(v)}, \\
\int_{U(v)} & =\frac{1}{C_{n(v)}^{3}} \sum_{s_{1}, s_{2}, s_{3}}\left[\int_{U_{s_{1}, s_{2}, s_{3}}(v)}+\int_{U(v)-U_{s_{1}, s_{2}, s_{3},(v)}}\right]
\end{aligned}
$$

where $n(v)$ is the valence of the vertex $v=s\left(s_{1}\right)=s\left(s_{2}\right)=s\left(s_{3}\right)$, and $C_{n}^{3} \equiv\left(\begin{array}{l}n \\ 3\end{array}\right)$ denotes the binomial coefficient. One then observes that

$$
\int_{U_{s_{1}, s_{2}, s_{3}}(v)}=8 \int_{\Delta_{s_{1}, s_{2}, s_{3}}^{0}(v)}
$$

in the limit $\epsilon \rightarrow 0$.

- The triangulation for the regions

$$
\begin{aligned}
& U(v)-U_{s_{1}, s_{2}, s_{3}}(v), \\
& U_{\gamma(s, c)}-\cup_{v \in V(\gamma(s, c))} U(v), \\
& \Sigma-U_{\gamma(s, c),}
\end{aligned}
$$

are arbitrary. These regions do not contribute to the construction of the operator, since the commutator terms like $\left[\hat{A}\left(s_{i}(\Delta)\right), \hat{V}_{U_{v(\Delta)}}\right] \Pi_{s, c}$ would vanish for all tetrahedron $\Delta$ in the regions (13).

Note that there are many possible ways in choosing a triangulation. The motivation for above choice has been fully discussed in Ref.[14]. Introducing a partition $\mathcal{P}$ of the 3 -manifold $\Sigma$ into cells $C$, we can smear the "square roots" of $\mathcal{H}_{K G, \phi}^{\epsilon}$ and $\mathcal{H}_{K G, K i n}^{\epsilon}$ in one cell $C$ respectively and promote them as regulated operators in $\mathcal{H}_{\text {kin }}$ with respect to the state-dependent triangulation $T(\epsilon)$ as

$$
\begin{aligned}
\hat{W}_{\gamma(s, c), \phi, i}^{\epsilon, C} & =\sum_{v \in V(\gamma(s, c))} \chi_{C}(v) \sum_{v(\Delta)=v} \frac{\hat{p}_{\Delta}}{\sqrt{\hat{E}(v)}} \hat{h}_{\phi, v, i}^{\epsilon, \Delta} \frac{\hat{p}_{\Delta}}{\sqrt{\hat{E}(v)}}, \\
\hat{W}_{\gamma(s, c), K i n}^{\epsilon, C} & =\sum_{v \in V(\gamma(s, c))} \chi_{C}(v) \sum_{v(\Delta)=v} \frac{\hat{p}_{\Delta}}{\sqrt{\hat{E}(v)}} \hat{h}_{K i n, v}^{\epsilon, \Delta} \frac{\hat{p}_{\Delta}}{\sqrt{\hat{E}(v)}},
\end{aligned}
$$


where $\chi_{C}(v)$ is the characteristic function of the cell $C$, and

$$
\begin{aligned}
\hat{h}_{\phi, v, i}^{\epsilon, \Delta}:= & \frac{i}{\hbar^{2}} \epsilon^{l m n} \frac{1}{\lambda(v)} \hat{U}(v, \lambda(v))^{-1}\left[\hat{U}\left(t\left(s_{l}(\Delta)\right), \lambda(v)\right)-\hat{U}(v, \lambda(v))\right] \times \\
& \operatorname{Tr}\left(\tau_{i} \hat{A}\left(s_{m}(\Delta)\right)^{-1}\left[\hat{A}\left(s_{m}(\Delta)\right), \hat{V}_{U_{v}^{\epsilon}}^{3 / 4}\right] \hat{A}\left(s_{n}(\Delta)\right)^{-1}\left[\hat{A}\left(s_{n}(\Delta)\right), \hat{V}_{U_{v}^{\epsilon}}^{3 / 4}\right]\right), \\
\hat{h}_{\text {Kin,v }}^{\epsilon, \Delta}:= & \frac{1}{(i \hbar)^{3}} \hat{\pi}(v) \epsilon^{l m n} \operatorname{Tr}\left(\hat{A}\left(s_{l}(\Delta)\right)^{-1}\left[\hat{A}\left(s_{l}(\Delta)\right), \sqrt{\hat{V}_{U_{v}^{\epsilon}}}\right] \times\right. \\
& \hat{A}\left(s_{m}(\Delta)\right)^{-1}\left[\hat{A}\left(s_{m}(\Delta)\right), \sqrt{\hat{V}_{U_{v}^{\epsilon}}}\right] \times \\
& \left.\hat{A}\left(s_{n}(\Delta)\right)^{-1}\left[\hat{A}\left(s_{n}(\Delta)\right), \sqrt{\hat{V}_{U_{v}^{\epsilon}}}\right]\right) .
\end{aligned}
$$

Note that the tetrahedron projector $\hat{p}_{\Delta}$ associated with segments $s_{1}, s_{2}$ and $s_{3}$ reads

$$
\begin{aligned}
\hat{p}_{\Delta} & :=\hat{p}_{s_{1}} \hat{p}_{s_{2}} \hat{p}_{s_{3}} \\
& =\theta\left(\sqrt{\frac{1}{4}-\Delta_{s_{1}}}-\frac{1}{2}\right) \theta\left(\sqrt{\frac{1}{4}-\Delta_{s_{2}}}-\frac{1}{2}\right) \theta\left(\sqrt{\frac{1}{4}-\Delta_{s_{3}}}-\frac{1}{2}\right)
\end{aligned}
$$

where $\Delta_{s_{i}}$ is the Casimir operator associated with the segment $s_{i}$ and $\theta$ is the distribution on $\mathbf{R}$ which vanishes on $(-\infty, 0]$ and equals 1 on $(0, \infty)$, and the tetrahedron projector related to a same vertex constitute the vertex operator $\hat{E}(v):=\sum_{v(\Delta)=v} \hat{p}_{\Delta}$. Note also that the partition $\mathcal{P}$ is not required to coincide with the triangulation $T(\epsilon)$. We have arranged the operator $\hat{p}_{\Delta} / \sqrt{\hat{E}(v)}$ in such a way that both operators in (14) and their adjoint operators are cylindrically consistent up to diffeomorphisms. Thus there are two densely defined operators $\hat{W}_{\phi, i}^{C}$ and $\hat{W}_{\text {Kin }}^{C}$ in $\mathcal{H}_{\text {kin }}$ associated with the two consistent families of (14). We now give several remarks on their properties.

\section{- Removal of regulator $\epsilon$}

It is not difficult to see that the action of the operator $\hat{W}_{\gamma(s, c), \phi, i}^{\epsilon, C}$ on a spin-scalarnetwork function $\Pi_{s, c}$ is graph-changing. It adds finite number of vertices with representation $\lambda(v)$ at $t\left(s_{i}(\Delta)\right)$ with distance $\epsilon$ from the vertex $v$. Recall that the action of the gravitational Hamiltonian constraint operator on a spin network function is also graph-changing. As a result, the family of operators $\hat{W}_{\gamma(s, c), \phi, i}^{\epsilon, C}$ also fails to be weakly convergent when $\epsilon \rightarrow 0$. However, due to the diffeomorphism covariant properties of the triangulation, the limit operator can be well defined via the uniform Rovelli-Smolin topology, or equivalently, the operator can be dually defined on diffeomorphism invariant states. But the dual operator cannot leave $\mathcal{H}_{\text {Diff }}$ invariant.

\section{- Quantization ambiguity}

As a main difference of the dynamics in polymer-like representation from that in $\mathrm{U}(1)$ group representation [22], a continuous label $\lambda$ appears explicitly in the expression of (14). Hence there is an one-parameter quantization ambiguity due to the real scalar field. Recall that the construction of gravitational Hamiltonian constraint operator also has a similar ambiguity due to the choice of the representations $j$ of the edges added by its action. A related quantization ambiguity 
also appears in the dynamics of loop quantum cosmology [26]. We will come back to this point in a future publication [27]. Since the regulator is removed in a diffeomorphism invariant way, the quadratic form, which we are going to constructed, will be independent of the initial triangulation $T$ in the sense that it depends only on the diffeomorphism class of $T$, as is the case of the gravitational Hamiltonian constraint operator [14].

Since our quantum field theory is expected to be diffeomorphism invariant, we would like to define the Hamiltonian operator of polymer scalar field in the diffeomorphism invariant Hilbert space $\mathcal{H}_{\text {Diff }}$. For this purpose we fix the parameter $\lambda$ to be a nonzero constant at every point. Then what we will do is to employ the new quantization strategy developed in Refs. [16] and [17]. We first construct a quadratic form in the light of a new inner product defined in Ref.[17] on the algebraic dual $\mathcal{D}^{\star}$ of the space of cylindrical functions. Then we prove that the quadratic form is closed. Note that, although the calculation by employing this inner product is formal, it can led to a welldefined expression of the desired quadratic form Eq. 22. Since an arbitrary element of $\mathcal{D}^{\star}$ is of the form $\Psi=\sum_{s, c} c_{s, c}<\Pi_{s, c} \mid \cdot>_{k i n}$, one can formally define an inner product $<\cdot|\cdot\rangle_{\star}$ on $\mathcal{D}^{\star}$ via

$$
\begin{aligned}
<\Psi, \Psi^{\prime}>_{\star} & :=\quad\left\langle\sum_{s, c} c_{s, c}<\Pi_{s, c}\left|\cdot>_{k i n}\right| \sum_{s^{\prime}, c^{\prime}} c_{s^{\prime}, c^{\prime}}^{\prime}<\Pi_{s^{\prime}, c^{\prime}}\right| \cdot>_{k i n}>_{\star} \\
& :=\sum_{s, c ; s^{\prime}, c^{\prime}} c_{s, c} \overline{c_{s^{\prime}, c^{\prime}}^{\prime}}<\Pi_{s, c} \mid \Pi_{s^{\prime}, c^{\prime}}>_{k i n} \frac{1}{\sqrt{\boldsymbol{\kappa}([s, c]) \boldsymbol{N}\left(\left[s^{\prime}, c^{\prime}\right]\right)}} \\
& =\sum_{s, c} c_{s, c} \overline{c_{s, c}^{\prime}} \frac{1}{\boldsymbol{\kappa}([s, c])},
\end{aligned}
$$

where the Cantor aleph $\boldsymbol{\aleph}$ denotes the cardinal of the set $[s, c]$. Note that we exchange the coefficients on which the complex conjugate was taken in Ref.[17], so that the inner product $<\Psi_{\text {Diff }} \mid \Psi_{D i f f}^{\prime}>_{\star}$ reduces to $<\Psi_{\text {Diff }} \mid \Psi_{\text {Diff }}^{\prime}>_{\text {Diff }}$ for any $\Psi_{\text {Diff }}, \Psi_{\text {Diff }}^{\prime} \in$ $\mathcal{H}_{\text {Diff }}$. Completing the quotient with respect to the null vectors by this inner product, one gets a Hilbert space $\mathcal{H}_{\star}$. Our purpose is to construct a quadratic form associated to some positive and symmetric operator in analogy with the classical expression of (12). So the quadratic form should first be given in a positive and symmetric version. It is then natural to define two quadratic forms on a dense subset of $\mathcal{H}_{\text {Diff }} \subset \mathcal{H}_{\star}$ as:

$$
\begin{gathered}
Q_{K G, \phi}\left(\Psi_{D i f f}, \Psi_{D i f f}^{\prime}\right):=\lim _{\mathcal{P} \rightarrow \Sigma} \sum_{C \in \mathcal{P}} 64 \times \frac{4 \alpha_{M}}{9 \kappa^{4} \gamma^{4}} \delta^{i j}<\hat{W}_{\phi, i}^{\prime C} \Psi_{D i f f} \mid \hat{W}_{\phi, j}^{\prime C} \Psi_{\text {Diff }}^{\prime}>_{\star}, \\
Q_{K G, \text { Kin }}\left(\Psi_{D i f f}, \Psi_{D i f f}^{\prime}\right):=\lim _{\mathcal{P} \rightarrow \Sigma} \sum_{C \in \mathcal{P}} 8^{4} \times \frac{16}{81 \alpha_{M}(\kappa \gamma)^{6}}<\hat{W}_{\text {Kin }}^{\prime C} \Psi_{\text {Diff }} \mid \hat{W}_{\text {Kin }}^{\prime C} \Psi_{\text {Diff }}^{\prime}>_{\star},
\end{gathered}
$$

where the dual limit operator $\hat{W}^{\prime C}$ of either family of $\hat{W}_{\phi, i}^{\epsilon, C}$ or $\hat{W}_{\text {Kin }}^{\epsilon, C}$ in 14 is naturally defined on diffeomorphism invariant states as

$$
\hat{W}^{\prime C} \Psi_{D i f f}\left[\Pi_{s, c}\right]=\lim _{\epsilon \rightarrow 0} \Psi_{D i f f}\left[\hat{W}^{\epsilon, C} \Pi_{s, c}\right] .
$$


To show that the quadratic forms are well defined, we write

$$
\begin{gathered}
\hat{W}_{\phi, i}^{\prime C} \Psi_{D i f f}=\sum_{s, c} w_{\phi, i, s, c}^{\Psi}(C)<\Pi_{s, c} \cdot>_{\star} \quad \Rightarrow \quad w_{\phi, i, s, c}^{\Psi}(C)=\left(\hat{W}_{\phi, i}^{\prime C} \Psi_{D i f f}\right)\left[\Pi_{s, c}\right], \\
\hat{W}_{\text {Kin }}^{\prime C} \Psi_{D i f f}=\sum_{s, c} w_{K i n, s, c}^{\Psi}(C)<\Pi_{s, c} \cdot>_{\star} \quad \Rightarrow \quad w_{\text {Kin, }, c, c}^{\Psi}(C)=\left(\hat{W}_{\text {Kin }}^{\prime C} \Psi_{D i f f}\right)\left[\Pi_{s, c}\right] .
\end{gathered}
$$

Then, by using the inner product (17) the quadratic forms in (18) become

$$
\begin{aligned}
& Q_{K G, \phi}\left(\Psi_{D i f f}, \Psi_{D i f f}^{\prime}\right) \\
:= & \lim _{\mathcal{P} \rightarrow \Sigma} \sum_{C \in \mathcal{P}} 64 \times \frac{4 \alpha_{M}}{9 \kappa^{4} \gamma^{4}} \delta^{i j} \sum_{s, c} w_{\phi, i, s, c}^{\Psi}(C) \overline{w_{\phi, j, s, c}^{\Psi \prime}(C)} \frac{1}{\boldsymbol{\aleph}([s, c])} \\
= & \lim _{\mathcal{P} \rightarrow \Sigma} \sum_{C \in \mathcal{P}} 64 \times \frac{4 \alpha_{M}}{9 \kappa^{4} \gamma^{4}} \delta^{i j} \sum_{[s, c]} \frac{1}{\boldsymbol{\aleph}([s, c])} \sum_{s, c \in[s, c]} w_{\phi, i, s, c}^{\Psi}(C) \overline{w_{\phi, j, s, c}^{\Psi \prime}(C)}, \\
& \frac{Q_{K G, K i n}\left(\Psi_{D i f f}, \Psi_{D i f f}^{\prime}\right)}{:=} \lim _{\mathcal{P} \rightarrow \Sigma} \sum_{C \in \mathcal{P}} 8^{4} \times \frac{16}{81 \alpha_{M}(\kappa \gamma)^{6}} \sum_{s, c} w_{K i n, s, c}^{\Psi}(C) \overline{w_{K i n, s, c}^{\Psi \prime}(C)} \frac{1}{\boldsymbol{\kappa ( [ s , c ] )}} \\
= & \lim _{\mathcal{P} \rightarrow \Sigma} \sum_{C \in \mathcal{P}} 8^{4} \times \frac{16}{81 \alpha_{M}(\kappa \gamma)^{6}} \sum_{[s, c]} \frac{1}{\boldsymbol{\aleph}([s, c])} \sum_{s, c \in[s, c]} w_{K i n, s, c}^{\Psi}(C) \overline{w_{K i n, s, c}^{\Psi^{\prime}}(C)} .
\end{aligned}
$$

Note that, since $\Psi_{\text {Diff }}$ is a finite linear combination of the diffeomorphism invariant spin-scalar-network basis, taking account of the operational property of $\hat{W}^{\prime C}$ there are only finite number of terms in the summation $\sum_{[s, c]}$ contributing to 20 . Hence we can interchange $\sum_{[s, c]}$ and $\lim _{\mathcal{P} \rightarrow \Sigma} \sum_{C \in \mathcal{P}}$ in above calculation. Moreover, for a sufficiently fine partition such that each cell contains at most one vertex, the sum over cells therefore reduces to finite terms with respect to the vertices of $\gamma(s, c)$. So we can interchange $\sum_{s, c \in[s, c]}$ and $\lim _{\mathcal{P} \rightarrow \Sigma} \sum_{C \in \mathcal{P}}$ to obtain:

$$
\begin{aligned}
& Q_{K G, \phi}\left(\Psi_{D i f f}, \Psi_{D i f f}^{\prime}\right) \\
& =64 \times \frac{4 \alpha_{M}}{9 \kappa^{4} \gamma^{4}} \delta^{i j} \sum_{[s, c]} \frac{1}{\aleph([s, c])} \sum_{s, c \in[s, c]} \lim _{\mathcal{P} \rightarrow \Sigma} \sum_{C \in \mathcal{P}} w_{\phi, i, s, c}^{\Psi}(C) \overline{w_{\phi, j, s, c}^{\Psi^{\prime}}(C)} \\
& =64 \times \frac{4 \alpha_{M}}{9 \kappa^{4} \gamma^{4}} \delta^{i j} \sum_{[s, c]} \frac{1}{\boldsymbol{\kappa}([s, c])} \sum_{s, c \in[s, c]} \sum_{v \in V(\gamma(s, c))}\left(\hat{W}_{\phi, i}^{\prime \prime} \Psi_{D i f f}\right)\left[\Pi_{s, c}\right] \overline{\left(\hat{W}_{\phi, j}^{\prime \prime} \Psi_{D i f f}^{\prime}\right)\left[\Pi_{s, c}\right]}, \\
& Q_{K G, K i n}\left(\Psi_{D i f f}, \Psi_{D i f f}^{\prime}\right) \\
& =8^{4} \times \frac{16}{81 \alpha_{M}(\kappa \gamma)^{6}} \sum_{[s, c]} \frac{1}{\aleph([s, c])} \sum_{s, c \in[s, c]} \lim _{\mathcal{P} \rightarrow \Sigma} \sum_{C \in \mathcal{P}} w_{K i n, s, c}^{\Psi}(C) \overline{w_{K i n, s, c}^{\Psi^{\prime \prime}}(C)} \\
& =8^{4} \times \frac{16}{81 \alpha_{M}(\kappa \gamma)^{6}} \sum_{[s, c]} \frac{1}{\boldsymbol{\aleph}([s, c])} \sum_{s, c \in[s, c]} \sum_{v \in V(\gamma(s, c))}\left(\hat{W}_{K i n}^{\prime \prime} \Psi_{D i f f}\right)\left[\Pi_{s, c}\right] \overline{\left(\hat{W}_{K i n}^{\prime v} \Psi_{D i f f}^{\prime}\right)\left[\Pi_{s, c}\right]},
\end{aligned}
$$


where the limit $\mathcal{P} \rightarrow \Sigma$ has been taken so that $C \rightarrow v$. Since given $\gamma(s, c)$ and $\gamma\left(s^{\prime}, c^{\prime}\right)$ which are different up to a diffeomorphism transformation, there is always a diffeomorphism $\varphi$ transforming the graph associated with $\hat{W}_{\gamma(s, c)}^{\epsilon, v} \Pi_{s, c}(v \in \gamma(s, c))$ to that of $\hat{W}_{\gamma\left(s^{\prime}, c^{\prime}\right)}^{\epsilon, \nu^{\prime}} \Pi_{s^{\prime}, c^{\prime}}\left(v^{\prime} \in \gamma\left(s^{\prime}, c^{\prime}\right)\right)$ with $\varphi(v)=v^{\prime},\left(\hat{W}^{\prime v} \Psi_{D i f f}\right)\left[\Pi_{s, c \in[s, c]}\right]$ is constant for different $(s, c) \in[s, c]$, i.e., all the $\boldsymbol{\kappa}([s, c])$ terms in the sum over $(s, c) \in[s, c]$ are identical. Hence the final expressions of the two quadratic forms can be written as:

$$
\begin{aligned}
& Q_{K G, \phi}\left(\Psi_{D i f f}, \Psi_{D i f f}^{\prime}\right) \\
= & 64 \times \frac{4 \alpha_{M}}{9 \kappa^{4} \gamma^{4}} \delta^{i j} \sum_{[s, c]} \sum_{v \in V(\gamma(s, c))}\left(\hat{W}_{\phi, i}^{\prime v} \Psi_{D i f f}\right)\left[\Pi_{s, c \in[s, c]}\right] \overline{\left(\hat{W}_{\phi, j}^{\prime v} \Psi_{D i f f}^{\prime}\right)\left[\Pi_{s, c \in[s, c]}\right]}, \\
& Q_{K G, K i n}\left(\Psi_{D i f f}, \Psi_{D i f f}^{\prime}\right) \\
= & 8^{4} \times \frac{16}{81 \alpha_{M}(\kappa \gamma)^{6}} \sum_{[s, c]} \sum_{v \in V(\gamma(s, c))}\left(\hat{W}_{K i n}^{\prime v} \Psi_{D i f f}\right)\left[\Pi_{s, c \in[s, c]}\right] \overline{\left(\hat{W}_{K i n}^{\prime v} \Psi_{D i f f}^{\prime}\right)\left[\Pi_{s, c \in[s, c]}\right]} .
\end{aligned}
$$

Note that both quadratic forms in (22) have finite results and hence their form domains are dense in $\mathcal{H}_{\text {Diff }}$. Moreover, both of them are obviously positive, and the following theorem will demonstrate their closedness.

Theorem: Both $Q_{K G, \phi}$ and $Q_{K G, K i n}$ are densely defined, positive and closed quadratic forms on $\mathcal{H}_{\text {Diff }}$, which are associated uniquely with two positive self-adjoint operators respectively on $\mathcal{H}_{\text {Diff }}$ such that

$$
\begin{aligned}
& Q_{K G, \phi}\left(\Psi_{D i f f}, \Psi_{D i f f}^{\prime}\right)=<\Psi_{D i f f}\left|\hat{\mathcal{H}}_{K G, \phi}\right| \Psi_{D i f f}^{\prime}>_{D i f f} \\
& Q_{K G, \text { Kin }}\left(\Psi_{\text {Diff }}, \Psi_{\text {Diff }}^{\prime}\right)=<\Psi_{\text {Diff }}\left|\hat{\mathcal{H}}_{K G, K i n}\right| \Psi_{\text {Diff }}^{\prime}>_{\text {Diff }} .
\end{aligned}
$$

Therefore the Hamiltonian operator

$$
\hat{\mathcal{H}}_{K G}:=\hat{\mathcal{H}}_{K G, \phi}+\hat{\mathcal{H}}_{K G, K i n}
$$

is positive and also have a unique self-adjoint extension.

Proof: We follow the strategy developed in Refs.[17] and [18] to prove that both $Q_{K G, \phi}$ and $Q_{K G, K i n}$ are closeable and uniquely induce two positive self-adjoint operators $\hat{\mathcal{H}}_{K G, \phi}$ and $\hat{\mathcal{H}}_{K G, \text { Kin }}$. One can formally define $\hat{\mathcal{H}}_{K G, \phi}$ and $\hat{\mathcal{H}}_{K G, \text { Kin }}$ acting on diffeomorphism invariant spin-scalar network functions via:

$$
\begin{aligned}
\hat{\mathcal{H}}_{K G, \phi} \Pi_{\left[s_{1}, c_{1}\right]} & :=\sum_{\left[s_{2}, c_{2}\right]} Q_{K G, \phi}\left(\Pi_{\left[s_{2}, c_{2}\right]}, \Pi_{\left[s_{1}, c_{1}\right]}\right) \Pi_{\left[s_{2}, c_{2}\right]}, \\
\hat{\mathcal{H}}_{K G, \text { Kin }} \Pi_{\left[s_{1}, c_{1}\right]} & :=\sum_{\left[s_{2}, c_{2}\right]} Q_{K G, K i n}\left(\Pi_{\left[s_{2}, c_{2}\right]}, \Pi_{\left[s_{1}, c_{1}\right]}\right) \Pi_{\left[s_{2}, c_{2}\right]} .
\end{aligned}
$$

Then we need to show that both of the above operators are densely defined on the Hilbert space $\mathcal{H}_{\text {Diff }}$, i.e.,

$$
\left\|\hat{\mathcal{H}}_{K G, \phi} \Pi_{\left[s_{1}, c_{1}\right]}\right\|_{D i f f}=\sum_{\left[s_{2}, c_{2}\right]}\left|Q_{K G, \phi}\left(\Pi_{\left[s_{2}, c_{2}\right]}, \Pi_{\left[s_{1}, c_{1}\right]}\right)\right|^{2}<\infty
$$




$$
\left\|\hat{\mathcal{H}}_{K G, K i n} \Pi_{\left[s_{1}, c_{1}\right]}\right\|_{D i f f}=\sum_{\left[s_{2}, c_{2}\right]}\left|Q_{K G, K i n}\left(\Pi_{\left[s_{2}, c_{2}\right]}, \Pi_{\left[s_{1}, c_{1}\right]}\right)\right|^{2}<\infty .
$$

Given a diffeomorphism invariant spin-scalar network function $\Pi_{\left[s_{1}, c_{1}\right]}$, there are only finite number of terms $\Pi_{\left[s_{1}, c_{1}\right]}\left[\hat{W}_{\gamma(s, c)}^{\epsilon, v} \Pi_{s, c \in[s, c]}\right]$ which are nonzero in the sum over equivalent classes $[s, c]$ in (22). On the other hand, given one spin-scalar network function $\Pi_{s, c \in[s, c]}$, there are also only finite number of possible $\Pi_{\left[s_{2}, c_{2}\right]}$ such that the terms $\overline{\Pi_{\left[s_{2}, c_{2}\right]}\left[\hat{W}_{\gamma(s, c)}^{\epsilon, v} \Pi_{s, c \in[s, c]}\right]}$ are nonzero. As a result, only finite number of terms survive in both sums over $\left[s_{2}, c_{2}\right]$ in Eqs. (26) and (27). Hence both $\hat{\mathcal{H}}_{K G, \phi}$ and $\hat{\mathcal{H}}_{K G, K i n}$ are well defined. Then it follows from Eqs. (22), (24) and (25) that they are positive and symmetric operators densely defined in $\mathcal{H}_{\text {Diff }}$, whose quadratic forms coincide with $Q_{K G, \phi}$ and $Q_{K G, K i n}$ on their form domains. Hence both $Q_{K G, \phi}$ and $Q_{K G, K i n}$ have positive closures and uniquely induce self-adjoint (Friedrichs) extensions of $\hat{\mathcal{H}}_{K G, \phi}$ and $\hat{\mathcal{H}}_{K G, \text { Kin }}$ respectively [28], which we denote by $\hat{\mathcal{H}}_{K G, \phi}$ and $\hat{\mathcal{H}}_{K G, K i n}$ as well. As a result, the Hamiltonian operator $\hat{\mathcal{H}}_{K G}$ defined by Eq.(23) is also positive and symmetric. Hence it has a unique self-adjoint (Friedrichs) extension.

口

We notice that, from a different perspective, one can construct the same Hamiltonian operator $\hat{\mathcal{H}}_{K G}$ without introducing an inner product on $\mathcal{D}^{\star}$. The construction is sketched as follows. Using the two well-defined operators $\hat{W}_{\phi, i}^{\epsilon, C}$ and $\hat{W}_{\text {Kin }}^{\epsilon, C}$ as in (14), as well as their adjoint operators $\left(\hat{W}_{\phi, i}^{\epsilon, C}\right)^{\dagger}$ and $\left(\hat{W}_{\text {Kin }}^{\epsilon, C}\right)^{\dagger}$, one may define two operators on $\mathcal{H}_{\text {Diff }}$ corresponding to the two terms in (12) by

$$
\begin{aligned}
\left(\hat{\mathcal{H}}_{K G, \phi} \Psi_{D i f f}\right)\left[f_{\gamma}\right] & =\lim _{\epsilon, \epsilon^{\prime} \rightarrow 0, \mathcal{P} \rightarrow \Sigma} \Psi_{D i f f}\left[\sum_{C \in \mathcal{P}} 64 \times \frac{4 \alpha_{M}}{9 \kappa^{4} \gamma^{4}} \delta^{i j} \hat{W}_{\phi, i}^{\epsilon, C}\left(\hat{W}_{\phi, j}^{\epsilon^{\prime}, C}\right)^{\dagger} f_{\gamma}\right] \\
\left(\hat{\mathcal{H}}_{K G, K i n} \Psi_{D i f f}\right)\left[f_{\gamma}\right] & =\lim _{\epsilon, \epsilon^{\prime} \rightarrow 0, \mathcal{P} \rightarrow \Sigma} \Psi_{D i f f}\left[\sum_{C \in \mathcal{P}} 8^{4} \times \frac{16}{81 \alpha_{M}(\kappa \gamma)^{6}} \hat{W}_{K i n}^{\epsilon, C}\left(\hat{W}_{K i n}^{\epsilon^{\prime}, C}\right)^{\dagger} f_{\gamma}\right] .
\end{aligned}
$$

In analogy with the discussion in section 5 and Ref.[18], it can be shown that both above operators leave $\mathcal{H}_{\text {Diff }}$ invariant and are densely defined on $\mathcal{H}_{\text {Diff }}$. Moreover, the quadratic forms associated with them coincide with the quadratic forms in 22). Thus the Hamiltonian operator $\hat{\mathcal{H}}_{K G}:=\hat{\mathcal{H}}_{K G, \phi}+\hat{\mathcal{H}}_{K G, K i n}$ coincides with the one constructed in the quadratic form approach.

In summary, we have constructed a positive self-adjoint Hamiltonian operator on $\mathcal{H}_{\text {Diff }}$ for the polymer-like scalar field, depending on a chosen parameter $\lambda$. Thus there is an 1-parameter ambiguity in the construction. However, there is no UV divergence in this quantum Hamiltonian without renormalization, since quantum gravity is presented as a natural regulator for the polymer-like scalar field.

\section{Quantum Hamiltonian constraint equation}

In this section we consider the whole dynamical system of scalar field coupled to gravity. Recall that in perturbative quantum field theory in curved spacetime, the definition 
of some basic physical quantities, such as the expectation value of energy-momentum, is ambiguous and it is extremely difficult to calculate the back-reaction of quantum fields to the background spacetime [29]. This is reflected by the fact that the semiclassical Einstein equation,

$$
R_{\alpha \beta}[g]-\frac{1}{2} R[g] g_{\alpha \beta}=\kappa<\hat{T}_{\alpha \beta}[g]>,
$$

is inconsistent and ambiguous [30][1]. One could speculate on that the difficulty is related to the fact that the present formulation of quantum field theories are background dependent. According to this speculation, if the quantization programme is by construction non-perturbative and background independent, it is possible to solve the problems fundamentally. In loop quantum gravity, there is no assumption of a priori background metric at all. The quantum geometry and quantum matter fields are coupled and fluctuating naturally with respect to each other on a common manifold. On the other hand, there exists the "time problem" in quantum theory of pure gravity, since all the physical states have to satisfy certain version of quantum Wheeler-DeWitt constraint equation. However, the situation would be improved when matter field is coupled to gravity. In the following construction, we impose the quantum Hamiltonian constraint on $\mathcal{H}_{\text {kin }}$, and thus define a quantum Wheeler-DeWitt constraint equation for the scalar field coupled to gravity. Then one can gain an insight into the problem of time from the coupled equation, and the back-reaction of the quantum scalar field is included in the framework of loop quantum gravity.

Recall that the gravitational Hamiltonian constraint operator $\hat{\mathcal{H}}_{G R}(N)$ can be well defined in $\mathcal{H}_{\text {kin }}^{G R}$ by the uniform Rovelli-Smolin topology [14]|[5]. Hence it is also well defined in the coupled kinematical Hilbert space $\mathcal{H}_{\text {kin }}$. Its regulated version via a statedependent triangulation $T(\epsilon)$ reads

$$
\begin{aligned}
\hat{\mathcal{H}}_{G R}^{\epsilon}(N)= & \hat{\mathcal{H}}_{E}^{\epsilon}(N)-2\left(1+\gamma^{2}\right) \hat{\mathcal{T}}^{\epsilon}(N) \\
\hat{\mathcal{H}}_{E, \alpha}^{\epsilon}(N)= & \frac{16}{3 i \hbar \kappa^{2} \gamma} \sum_{v \in V(\alpha)} N(v) \sum_{v(\Delta)=v} \epsilon^{i j k} \times \\
& \operatorname{Tr}\left(\hat{A}\left(\alpha_{i j}(\Delta)\right)^{-1} \hat{A}\left(s_{k}(\Delta)\right)^{-1}\left[\hat{A}\left(s_{k}(\Delta)\right), \hat{V}_{U_{v}^{\epsilon}}\right]\right) \frac{\hat{p}_{\Delta}}{\hat{E}(v)}, \\
\hat{\mathcal{T}}_{\alpha}^{\epsilon}(N)= & -\frac{4 \sqrt{2}}{3 i \hbar^{3} \kappa^{4} \gamma^{3}} \sum_{v \in V(\alpha)} N(v) \sum_{v(\Delta)=v} \epsilon^{i j k} \times \\
& \operatorname{Tr}\left(\hat{A}\left(s_{i}(\Delta)\right)^{-1}\left[\hat{A}\left(s_{i}(\Delta)\right), \hat{K}^{\epsilon}\right] \hat{A}\left(s_{j}(\Delta)\right)^{-1}\left[\hat{A}\left(s_{j}(\Delta)\right), \hat{K}^{\epsilon}\right] \times\right. \\
& \left.\hat{A}\left(s_{k}(\Delta)\right)^{-1}\left[\hat{A}\left(s_{k}(\Delta)\right), \hat{V}_{U_{v}^{\epsilon}}\right]\right) \frac{\hat{p}_{\Delta}}{\hat{E}(v)} .
\end{aligned}
$$

We now define an operator in $\mathcal{H}_{\text {kin }}$ corresponding to the scalar field part $\mathcal{H}_{K G}(N)$ of the total Hamiltonian constraint functional, which can be read out from Eqs. (1) and (4) as

$$
\mathcal{H}_{K G}(N)=\mathcal{H}_{K G, \phi}(N)+\mathcal{H}_{K G, \text { Kin }}(N),
$$

where

$$
\mathcal{H}_{K G, \phi}(N)=\frac{\kappa^{2} \gamma^{2} \alpha_{M}}{2} \int_{\Sigma} d^{3} x N \frac{1}{\sqrt{|\operatorname{det} q|}} \delta^{i j} \widetilde{P}_{i}^{a} \widetilde{P}_{j}^{b}\left(\partial_{a} \phi\right) \partial_{b} \phi
$$




$$
\mathcal{H}_{K G, \text { Kin }}(N)=\frac{1}{2 \alpha_{M}} \int_{\Sigma} d^{3} x N \frac{1}{\sqrt{|\operatorname{det} q|}} \widetilde{\pi}^{2} .
$$

In analogy with the regularization and quantization in the previous section, the regulated version of quantum Hamiltonian constraint $\hat{\mathcal{H}}_{K G}^{\epsilon}(N)$ of scalar field is expressed via a state-dependent triangulation $T(\epsilon)$ as:

$$
\hat{\mathcal{H}}_{K G, \gamma}^{\epsilon}(N):=\sum_{v \in V(\gamma)} N(v)\left[\delta^{i j}\left(\hat{W}_{\gamma, \phi, i}^{\epsilon, v}\right)^{\dagger} \hat{W}_{\gamma, \phi, j}^{\epsilon, v}+\left(\hat{W}_{\gamma, \text { Kin }}^{\epsilon, \nu}\right)^{\dagger} \hat{W}_{\gamma, \text { Kin }}^{\epsilon, v}\right]
$$

where the operators

$$
\begin{gathered}
\hat{W}_{\gamma, \phi, i}^{\epsilon, v}:=\sum_{v(\Delta)=v} \frac{\hat{p}_{\Delta}}{\sqrt{\hat{E}(v)}} \hat{h}_{\phi, v, i}^{\epsilon, \Delta} \frac{\hat{p}_{\Delta}}{\sqrt{\hat{E}(v)}}, \quad \hat{W}_{\gamma, \text { Kin }}^{\epsilon, v}:=\sum_{v(\Delta)=v} \frac{\hat{p}_{\Delta}}{\sqrt{\hat{E}(v)}} \hat{h}_{K i n, v}^{\epsilon, \Delta} \frac{\hat{p}_{\Delta}}{\sqrt{\hat{E}(v)}} \\
\left(\hat{W}_{\gamma, \phi, i}^{\epsilon, v}\right)^{\dagger}:=\sum_{v(\Delta)=v} \frac{\hat{p}_{\Delta}}{\sqrt{\hat{E}(v)}}\left(\hat{h}_{\phi, v, i}^{\epsilon, \Delta}\right)^{\dagger} \frac{\hat{p}_{\Delta}}{\sqrt{\hat{E}(v)}} \quad, \quad\left(\hat{W}_{\gamma, \text { Kin }}^{\epsilon, v}\right)^{\dagger}:=\sum_{v(\Delta)=v} \frac{\hat{p}_{\Delta}}{\sqrt{\hat{E}(v)}}\left(\hat{h}_{K i n, v}^{\epsilon, \Delta}\right)^{\dagger} \frac{\hat{p}_{\Delta}}{\sqrt{\hat{E}(v)}}
\end{gathered}
$$

are all cylindrically consistent up to diffeomorphisms. Hence the family of Hamiltonian constraint operators (31) is also cylindrically consistent up to diffeomorphisms, and the regulator $\epsilon$ can be removed via the uniform Rovelli-Smollin topology, or equivalently the limit operator dually acts on diffeomorphism invariant states as

$$
\left(\hat{\mathcal{H}}_{K G}^{\prime}(N) \Psi_{D i f f}\right)\left[f_{\gamma}\right]=\lim _{\epsilon \rightarrow 0} \Psi_{D i f f}\left[\hat{\mathcal{H}}_{K G, \gamma}^{\epsilon}(N) f_{\gamma}\right],
$$

for any $f_{\gamma} \in C y l_{\gamma(s, c)}(\overline{\mathcal{A}}) \otimes C y l_{\gamma(s, c)}(\overline{\mathcal{U}})$. Similar to the dual of $\hat{\mathcal{H}}_{G R}(N)$, the operator $\hat{\mathcal{H}}_{K G}^{\prime}(N)$ fails to commute with the dual of finite diffeomorphism transformation operators, unless the smearing function $N(x)$ is a constant function over $\Sigma$. Note that the diffeomorphism invariant Hamiltonian operator $\hat{\mathcal{H}}_{K G}$ defined in the previous section is actually $\hat{\mathcal{H}}_{K G}^{\prime}(1)$. From Eq. (31), it is not difficult to prove that for positive $N(x)$ the Hamiltonian constraint operator $\hat{\mathcal{H}}_{K G}(N)$ of scalar field is positive and symmetric in $\mathcal{H}_{\text {kin }}$ and hence has a unique self-adjoint extension. It is pointed out in Ref.[31] that, there can be problems associated with symmetric constraint operators for systems where the constraints close with structure functions as is the present case. However, not all the assumptions underlying this conclusion are valid in the framework of loop quantum gravity. For example, it is assumed in Ref.[31] that all the classical canonical variables and constraints could be promoted as well-defined operators in the kinematical Hilbert space. However, it is well known that the classical connection and diffeomorphsim constraint cannot be represented as well-defined operators in loop quantum gravity. This issue related to the symmetric Hamiltonian constraint operator was fully discussed in Ref.[14].

Our construction of $\hat{\mathcal{H}}_{K G}(N)$ is similar to that of the Higgs field Hamiltonian constraint in Ref.[22]. However, like the case of $\hat{\mathcal{H}}_{K G}$, there is an 1-parameter ambiguity in our construction of $\hat{\mathcal{H}}_{K G}(N)$ due to the real scalar field, which is manifested as the continuous parameter $\lambda$ in the expression of $\hat{h}_{\phi, v, i}^{\epsilon, \Delta}$ in (15). Note that now $\lambda$ is not required to be a constant, i.e., its value can be changed from one point to another. This 
issue of ambiguity will be discussed again in a future publication [27]. Thus the total Hamiltonian constraint operator of scalar field coupled to gravity has been obtained as

$$
\hat{\mathcal{H}}(N)=\hat{\mathcal{H}}_{G R}(N)+\hat{\mathcal{H}}_{K G}(N)
$$

Again, there is no UV divergence in this quantum Hamiltonian constraint. Recall that, in standard quantum field theory the UV divergence can only be cured by renormalization procedure, in which one has to multiply the Hamiltonian by a suitable power of the regulating parameter $\epsilon$ artificially. While, now $\epsilon$ has naturally disappeared from the expressions of (23) and (33). So renormalization is not needed for the polymer-like scalar field coupled to gravity, since quantum gravity has been presented as a natural regulator. Together with the result in the previous section, this heightens our confidence that the issue of divergence in quantum fields theory can be cured in the framework of loop quantum gravity. The desired matter-coupled Wheeler-DeWitt equation can be well imposed as:

$$
-\left(\hat{\mathcal{H}}_{K G}^{\prime}(N) \Psi_{D i f f}\right)\left[f_{\gamma}\right]=\left(\hat{\mathcal{H}}_{G R}^{\prime}(N) \Psi_{D i f f}\right)\left[f_{\gamma}\right]
$$

Note that the scalar field part $\hat{\mathcal{H}}_{K G}(N)$ acts nontrivially on gravitational quantum states. This can be regarded as the "back-reaction" of quantum matter field to the quantum gravitational field. On the other hand, comparing Eq. (34) with the well-known Schördinger equation for a particle,

$$
i \hbar \frac{\partial}{\partial t} \psi(x, t)=H\left(\hat{x},-\overline{i \hbar \frac{\partial}{\partial x}}\right) \psi(x, t),
$$

where $\psi(x, t) \in L^{2}(\mathbf{R}, d x)$ and $t$ is a parameter labelling time evolution, one may take the viewpoint that the matter field constraint operator $\hat{\mathcal{H}}_{K G}^{\prime}(N)$ plays the role of $i \hbar \frac{\partial}{\partial t}$. Then $\phi$ appears as the parameter labelling the evolution of the gravitational field state. In the reverse viewpoint, gravitational field would become the parameter labelling the evolution of the quantum matter field.

\section{Master constraint programme}

In order to avoid possible quantum anomaly and find the physical Hilbert space of quantum gravity, master constraint programme was first introduced by Thiemann in [16]. The central idea is to construct an alternative classical constraint algebra, giving the same constraint phase space, which is a Lie algebra (no structure function) and where the subalgebra of diffeomorphism constraints forms an ideal. Self-adjoint master constraint operators for loop quantum gravity are then proposed in Refs. [17] and [18]. The master constraint programme can be generalized to matter fields coupled to gravity in a straightforward way. We now take the massless real scalar field to demonstrate the construction of a master constraint operator according to the strategy in Ref.[18]. By this approach one not only avoids possible quantum anomaly which might appear in the conventional canonical quantization method, but also might give a qualitative 
description of the physical Hilbert space for the coupled system. We introduce the master constraint for the scalar field coupled to gravity as

$$
\mathbf{M}:=\frac{1}{2} \int_{\Sigma} d^{3} x \frac{|C(x)|^{2}}{\sqrt{|\operatorname{det} q(x)|}},
$$

where $C(x)$ is the Hamiltonian constraint in (4). After solving the Gaussian constraint, one gets the master constraint algebra as a Lie algebra:

$$
\begin{aligned}
\left\{\mathcal{V}(\vec{N}), \mathcal{V}\left(\vec{N}^{\prime}\right)\right\} & =\mathcal{V}\left(\left[\vec{N}, \vec{N}^{\prime}\right]\right), \\
\{\mathcal{V}(\vec{N}), \mathbf{M}\} & =0 \\
\{\mathbf{M}, \mathbf{M}\} & =0
\end{aligned}
$$

where the subalgebra of diffeomorphism constraints forms an ideal. So it is possible to define a corresponding master constraint operator on $\mathcal{H}_{\text {Diff }}$. In the following, the positivity and the diffeomorphism invariance of $\mathbf{M}$ will be working together properly and provide us with powerful functional analytic tools in the quantization procedure.

The regulated version of the master constraint can be expressed via a point-splitting strategy as:

$$
\mathbf{M}^{\epsilon}:=\frac{1}{2} \int_{\Sigma} d^{3} y \int_{\Sigma} d^{3} x \chi_{\epsilon}(x-y) \frac{C(y)}{\sqrt{V_{U_{y}^{\epsilon}}}} \frac{C(x)}{\sqrt{V_{U_{x}^{\epsilon}}}} .
$$

Introducing a partition $\mathcal{P}$ of the 3-manifold $\Sigma$ into cells $C$, we have an operator $\hat{H}_{C, \gamma}^{\epsilon}$ acting on any cylindrical function $f_{\gamma} \in C y l_{\gamma}^{3}(\overline{\mathcal{A}}) \otimes C y l_{\gamma}(\overline{\mathcal{U}})$ via a state-dependent triangulation $T(\epsilon)$,

$$
\begin{aligned}
\hat{H}_{C, \gamma}^{\epsilon} & =\sum_{\nu \in V(\gamma)} \chi_{C}(v) \sum_{\nu(\Delta)=v} \frac{\hat{p}_{\Delta}}{\sqrt{\hat{E}(v)}} \hat{h}_{G R, v}^{\epsilon, \Delta} \frac{\hat{p}_{\Delta}}{\sqrt{\hat{E}(v)}} \\
& +\sum_{\nu \in V(\gamma)} \chi_{C}(v)\left[\delta^{i j}\left(\hat{w}_{\gamma, \phi, i}^{\epsilon, v}\right)^{\dagger} \hat{w}_{\gamma, \phi, j}^{\epsilon, v}+\left(\hat{w}_{\gamma, K i n}^{\epsilon, \nu}\right)^{\dagger} \hat{w}_{\gamma, K i n}^{\epsilon, \nu}\right],
\end{aligned}
$$

where

$$
\begin{aligned}
\hat{h}_{G R, v}^{\epsilon, \Delta} & =\frac{16}{3 i \hbar \kappa^{2} \gamma} \epsilon^{i j k} \operatorname{Tr}\left(\hat{A}\left(\alpha_{i j}(\Delta)\right)^{-1} \hat{A}\left(s_{k}(\Delta)\right)^{-1}\left[\hat{A}\left(s_{k}(\Delta)\right), \sqrt{\hat{V}_{U_{v}^{\epsilon}}}\right]\right) \\
& +\left(1+\gamma^{2}\right) \frac{8 \sqrt{2}}{3 i \hbar^{3} \kappa^{4} \gamma^{3}} \epsilon^{i j k} \operatorname{Tr}\left(\hat{A}\left(s_{i}(\Delta)\right)^{-1}\left[\hat{A}\left(s_{i}(\Delta)\right), \hat{K}^{\epsilon}\right]\right. \\
& \left.\times \hat{A}\left(s_{j}(\Delta)\right)^{-1}\left[\hat{A}\left(s_{j}(\Delta)\right), \hat{K}^{\epsilon}\right] \hat{A}\left(s_{k}(\Delta)\right)^{-1}\left[\hat{A}\left(s_{k}(\Delta)\right), \sqrt{\hat{V}_{U_{v}}}\right]\right), \\
\hat{w}_{\gamma, \phi, i}^{\epsilon, v} & =\frac{i}{\hbar^{2}} \sum_{v(\Delta)=v} \frac{\hat{p}_{\Delta}}{\sqrt{\hat{E}(v)}} \epsilon^{l m n} \frac{1}{\lambda} \hat{U}(v, \lambda)^{-1}\left[\hat{U}\left(t\left(s_{l}(\Delta)\right), \lambda\right)-\hat{U}(v, \lambda)\right] \\
& \times \operatorname{Tr}\left(\tau_{i} \hat{A}\left(s_{m}(\Delta)\right)^{-1}\left[\hat{A}\left(s_{m}(\Delta)\right), \hat{V}_{U_{v}^{\epsilon}}^{5 / 8}\right] \hat{A}\left(s_{n}(\Delta)\right)^{-1}\left[\hat{A}\left(s_{n}(\Delta)\right), \hat{V}_{U_{v}^{\epsilon}}^{5 / 8}\right]\right) \frac{\hat{p}_{\Delta}}{\sqrt{\hat{E}(v)}} \\
\hat{w}_{\gamma, \text { Kin }}^{\epsilon, v} & =\frac{1}{(i \hbar)^{3}} \sum_{v(\Delta)=v} \frac{\hat{p}_{\Delta}}{\sqrt{\hat{E}(v)}} \hat{\pi}(v) \epsilon^{l m n}
\end{aligned}
$$




$$
\begin{aligned}
& \times \operatorname{Tr}\left(\hat{A}\left(s_{l}(\Delta)\right)^{-1}\left[\hat{A}\left(s_{l}(\Delta)\right), \hat{V}_{U_{v}^{\epsilon}}^{5 / 12}\right] \hat{A}\left(s_{m}(\Delta)\right)^{-1}\left[\hat{A}\left(s_{m}(\Delta)\right), \hat{V}_{U_{v}^{\epsilon}}^{5 / 12}\right]\right. \\
& \left.\times \hat{A}\left(s_{n}(\Delta)\right)^{-1}\left[\hat{A}\left(s_{n}(\Delta)\right), \hat{V}_{U_{v}^{\epsilon}}^{5 / 12}\right]\right) \frac{\hat{p}_{\Delta}}{\sqrt{\hat{E}(v)}} .
\end{aligned}
$$

The notations here are as same as those in section 3. Note that $\hat{H}_{C, \gamma}^{\epsilon}$ is similar to the Hamiltonian constraint operator $\hat{\mathcal{H}}(1)$ defined in last section, but is now divided by the square root of volume operator. Hence the action of $\hat{H}_{C, \gamma}^{\epsilon}$ on a cylindrical function $f_{\gamma}$ adds analytical $\operatorname{arcs} a_{i j}(\Delta)$ with $\frac{1}{2}$-representation (or arbitrary chosen spin $j$ representation of $S U(2)$ if one uses non-fundamental representations to express the holonomies in (30) and points at $t\left(s_{i}(\Delta)\right)$ with representation $\lambda$ with respect to each vertex $v(\Delta)$ of $\gamma$. Thus, for each $\epsilon>0, \hat{H}_{C, \gamma}^{\epsilon}$ is a $S U(2)$ gauge invariant and diffeomorphism covariant operator defined on $C y l_{\gamma}^{3}(\overline{\mathcal{A}}) \otimes C y l_{\gamma}(\overline{\mathcal{U}})$. The family of such operators with respect to different graphs are cylindrically consistent up to diffeomorphisms. So the inductive limit operator $\hat{H}_{C}$ is densely defined on $\mathcal{H}_{\text {Kin }}$ by the uniform RovelliSmolin topology. Moreover, the adjoint operators of $\hat{H}_{C, \gamma}^{\epsilon}$, which are also cylindrically consistent up to diffeomorphisms, read

$$
\begin{aligned}
\left(\hat{H}_{C, \gamma}^{\epsilon}\right)^{\dagger} & =\sum_{v \in V(\gamma)} \chi_{C}(v) \sum_{v(\Delta)=v} \frac{\hat{p}_{\Delta}}{\sqrt{\hat{E}(v)}}\left(\hat{h}_{G R, v}^{\epsilon, \Delta}\right)^{\dagger} \frac{\hat{p}_{\Delta}}{\sqrt{\hat{E}(v)}} \\
& +\sum_{v \in V(\gamma)} \chi_{C}(v)\left[\delta^{i j}\left(\hat{w}_{\gamma, \phi, j}^{\epsilon, v}\right)^{\dagger} \hat{w}_{\gamma, \phi, i}^{\epsilon, v}+\left(\hat{w}_{\gamma, \text { Kin }}^{\epsilon, v}\right)^{\dagger} \hat{w}_{\gamma, \text { Kin }}^{\epsilon, v}\right] .
\end{aligned}
$$

The inductive limit operator of (40) is denoted by $\left(\hat{H}_{C}^{\epsilon}\right)^{\dagger}$, which is adjoint to $\hat{H}_{C}$ as

$$
\begin{aligned}
<g_{\gamma^{\prime}}, \hat{H}_{C} f_{\gamma}>_{k i n}= & <g_{\gamma^{\prime}}, \hat{H}_{C, \gamma} f_{\gamma}>_{k i n}=<\left(\hat{H}_{C, \gamma}\right)^{\dagger} g_{\gamma^{\prime}}, f_{\gamma}>_{k i n} \\
= & <\left(\hat{H}_{C}\right)^{\dagger} g_{\gamma^{\prime}}, f_{\gamma}>_{k i n}=<\left(\hat{H}_{C}\right)_{\gamma^{\prime}}^{\dagger} g_{\gamma^{\prime}}, f_{\gamma}>_{k i n} .
\end{aligned}
$$

Then a master constraint operator, $\hat{\mathbf{M}}$, in $\mathcal{H}_{\text {Diff }}$ can be defined as:

$$
\left(\hat{\mathbf{M}} \Psi_{D i f f}\right)\left[\Pi_{s, c}\right]:=\lim _{\mathcal{P} \rightarrow \Sigma ; \epsilon, \epsilon^{\prime} \rightarrow 0} \Psi_{D i f f}\left[\sum_{C \in \mathcal{P}} \frac{1}{2} \hat{H}_{C}^{\epsilon}\left(\hat{H}_{C}^{\epsilon^{\prime}}\right)^{\dagger} \Pi_{s, c}\right] .
$$

Since $\hat{H}_{C}^{\epsilon}\left(\hat{H}_{C}^{\epsilon^{\prime}}\right)^{\dagger} \Pi_{s, c}$ is a finite linear combination of spin-scalar-network functions on an extended graph with skeleton $\gamma$, the value of $\left(\hat{\mathbf{M}} \Psi_{D i f f}\right)\left[\Pi_{s, c}\right]$ is finite for a given $\Psi_{\text {Diff }} \in \mathcal{H}_{\text {Diff }}$. So $\hat{\mathbf{M}} \Psi_{\text {Diff }}$ is in the algebraic dual of the space of cylindrical functions. Moreover, we can show that it is diffeomorphism invariant. For any diffeomorphism transformation $\varphi$,

$$
\begin{aligned}
\left(\hat{U}_{\varphi}^{\prime} \hat{\mathbf{M}} \Psi_{D i f f}\right)\left[f_{\gamma}\right] & =\lim _{\mathcal{P} \rightarrow \Sigma ; \epsilon, \epsilon^{\prime} \rightarrow 0} \Psi_{D i f f}\left[\sum_{C \in \mathcal{P}} \frac{1}{2} \hat{H}_{C}^{\epsilon}\left(\hat{H}_{C}^{\epsilon^{\prime}}\right)^{\dagger} \hat{U}_{\varphi} f_{\gamma}\right] \\
& =\lim _{\mathcal{P} \rightarrow \Sigma ; \epsilon, \epsilon^{\prime} \rightarrow 0} \Psi_{D i f f}\left[\hat{U}_{\varphi} \sum_{C \in \mathcal{P}} \frac{1}{2} \hat{H}_{\varphi^{-1}(C)}^{\varphi^{-1}(\epsilon)}\left(\hat{H}_{\varphi^{-1}(C)}^{\varphi^{-1}\left(\epsilon^{\prime}\right)}\right)^{\dagger} f_{\gamma}\right] \\
& =\lim _{\mathcal{P} \rightarrow \Sigma ; \epsilon, \epsilon^{\prime} \rightarrow 0} \Psi_{D i f f}\left[\sum_{C \in \mathcal{P}} \frac{1}{2} \hat{H}_{C}^{\epsilon}\left(\hat{H}_{C}^{\epsilon^{\prime}}\right)^{\dagger} f_{\gamma}\right],
\end{aligned}
$$


for any cylindrical function $f_{\gamma}$, where in the last step, we used the fact that the diffeomorphism transformation $\varphi$ leaves the partition invariant in the limit $\mathcal{P} \rightarrow \Sigma$ and relabel $\varphi(C)$ to be $C$. So we have the result

$$
\left(\hat{U}_{\varphi}^{\prime} \hat{\mathbf{M}} \Psi_{D i f f}\right)\left[f_{\gamma}\right]=\left(\hat{\mathbf{M}} \Psi_{D i f f}\right)\left[f_{\gamma}\right] .
$$

On the other hand, given any diffeomorphism invariant spin-scalar-network state $\Pi_{[s, c]}$, the norm of the result state $\hat{\mathbf{M}} \Pi_{[s, c]}$ can be expressed as:

$$
\begin{aligned}
& \mid \hat{\mathbf{M}}_{[s, c]} \|_{D i f f} \\
= & \sum_{\left[s^{\prime}, c^{\prime}\right]}\left|<\hat{\mathbf{M}}_{[s, c]}\right| \Pi_{\left[s^{\prime}, c^{\prime}\right]}>\left._{D i f f}\right|^{2} \\
= & \left.\left.\sum_{\left[s^{\prime}, c^{\prime}\right]}\right|_{\mathcal{P} \rightarrow \Sigma ; \epsilon, \epsilon^{\prime} \rightarrow 0} \prod_{[s, c]}\left[\sum_{C \in \mathcal{P}} \frac{1}{2} \hat{H}_{C}^{\epsilon}\left(\hat{H}_{C}^{\epsilon^{\prime}}\right)^{\dagger} \Pi_{s^{\prime}, c^{\prime} \in\left[s^{\prime}, c^{\prime}\right]}\right]\right|^{2} \\
= & \left.\sum_{\left[s^{\prime}, c^{\prime}\right]}\right|_{\mathcal{P} \rightarrow \Sigma ; \epsilon, \epsilon^{\prime} \rightarrow 0} \frac{1}{n_{\gamma(s, c)}} \sum_{\varphi \in D i f f / D i f f_{\gamma(s, c)}} \sum_{\varphi^{\prime} \in G S_{\gamma(s, c)}} \\
\times & <\hat{U}_{\varphi} \hat{U}_{\varphi^{\prime}} \Pi_{s, c \in[s, c]}\left|\sum_{C \in \mathcal{P}} \frac{1}{2} \hat{H}_{C}^{\epsilon}\left(\hat{H}_{C}^{\epsilon^{\prime}}\right)^{\dagger} \Pi_{s^{\prime}, c^{\prime} \in\left[s^{\prime}, c^{\prime}\right]}>_{K i n}\right|^{2} \\
= & \left.\sum_{\left[s^{\prime}, c^{\prime}\right]}\right|_{\mathcal{P} \rightarrow \Sigma ; \epsilon, \epsilon^{\prime} \rightarrow 0} \frac{1}{n_{\gamma(s, c)}} \sum_{\varphi \in D i f f / D i f f_{\gamma(s, c)}} \sum_{\varphi^{\prime} \in G S_{\gamma(s, c)}} \\
\times & <\hat{U}_{\varphi} \hat{U}_{\varphi^{\prime}} \sum_{C \in \mathcal{P}} \frac{1}{2} \hat{H}_{C}^{\epsilon^{\prime}}\left(\hat{H}_{C}^{\epsilon}\right)^{\dagger} \Pi_{s, c \in[s, c]} \Pi_{s^{\prime}, c^{\prime} \in\left[s^{\prime}, c^{\prime}\right]}>\left._{K i n}\right|^{2} \\
= & \left.\sum_{\left[s^{\prime}, c^{\prime}\right]}\right|_{\mathcal{P} \rightarrow \Sigma ; \epsilon, \epsilon^{\prime} \rightarrow 0} \prod_{\left[s^{\prime}, c^{\prime}\right]}\left[\left.\sum_{C \in \mathcal{P}} \frac{1}{2} \hat{H}_{C}^{\epsilon^{\prime}}\left(\hat{H}_{C}^{\epsilon}\right)^{\dagger} \Pi_{s, c \in[s, c]}\right|^{2},\right.
\end{aligned}
$$

where we make use of the fact that $\hat{\mathbf{M}}$ commutes with diffeomorphism transformations. Note that, the cylindrical function $\sum_{C \in \mathcal{P}} \frac{1}{2} \hat{H}_{C}^{\epsilon^{\prime}}\left(\hat{H}_{C}^{\epsilon}\right)^{\dagger} \Pi_{s, c \in[s, c]}$ is a finite linear combination of spin-scalar-network functions on some extended graph, so that there are only finite number of terms contributing to the sum in Eq. 455. Hence it automatically converges. So the master constraint operator $\hat{\mathbf{M}}$ defined by Eq.(42) is densely defined on $\mathcal{H}_{\text {Diff }}$.

We now compute the matrix elements of $\hat{\mathbf{M}}$. Given two diffeomorphism invariant spin-scalar-network functions $\Pi_{\left[s_{1}, c_{1}\right]}$ and $\Pi_{\left[s_{2}, c_{2}\right]}$, the matrix element of $\hat{\mathbf{M}}$ is calculated as

$$
\begin{aligned}
& \frac{<\Pi_{\left[s_{1}, c_{1}\right]}|\hat{\mathbf{M}}| \Pi_{\left[s_{2}, c_{2}\right]}>_{D i f f}}{\left(\hat{\mathbf{M}} \Pi_{\left[s_{2}, c_{2}\right]}\right]\left[\Pi_{s_{1}, c_{1} \in\left[s_{1}, c_{1}\right]}\right]} \\
= & \lim _{\mathcal{P} \rightarrow \Sigma ; \epsilon, \epsilon^{\prime} \rightarrow 0} \sum_{C \in \mathcal{P}} \frac{1}{2} \frac{\Pi_{\left[s_{2}, c_{2}\right]}\left[\hat{H}_{C}^{\epsilon}\left(\hat{H}_{C}^{\epsilon^{\prime}}\right)^{\dagger} \Pi_{s_{1}, c_{1} \in\left[s_{1}, c_{1}\right]}\right]}{\sum_{\mathcal{P} \rightarrow \Sigma ; \epsilon, \epsilon^{\prime} \rightarrow 0}} \sum_{C \in \mathcal{P}} \frac{1}{2} \frac{1}{n_{\gamma\left(s_{2}, c_{2}\right)}} \sum_{\varphi \in \text { Diff/Diff } f_{\gamma\left(s_{2}, c_{2}\right)} \sum_{\varphi^{\prime} \in G S_{\gamma\left(s_{2}, c_{2}\right)}}}
\end{aligned}
$$




$$
\begin{aligned}
& \times \overline{<\hat{U}_{\varphi} \hat{U}_{\varphi^{\prime}} \Pi_{s_{2}, c_{2} \in\left[s_{2}, c_{2}\right]} \mid \hat{H}_{C}^{\epsilon}\left(\hat{H}_{C}^{\epsilon^{\prime}}\right)^{\dagger} \Pi_{s_{1}, c_{2} \in\left[s_{1}, c_{1}\right]}>_{K i n}} \\
& =\frac{\sum_{s, c} \lim _{\mathcal{P} \rightarrow \Sigma ; \epsilon, \epsilon^{\prime} \rightarrow 0} \sum_{C \in \mathcal{P}} \frac{1}{2} \frac{1}{n_{\gamma\left(s_{2}, c_{2}\right)}} \sum_{\varphi \in \operatorname{Diff} / D i f f_{\gamma\left(s_{2}, c_{2}\right)}} \sum_{\varphi^{\prime} \in G S_{\gamma\left(s_{2}, c_{2}\right)}}}{<\hat{U}_{\varphi} \hat{U}_{\varphi^{\prime}} \Pi_{s_{2}, c_{2} \in\left[s_{2}, c_{2}\right]}\left|\hat{H}_{C, \gamma(s, c)}^{\epsilon} \Pi_{s, c}>_{K i n}<\Pi_{s, c}\right|\left(\hat{H}_{C}^{\epsilon^{\prime}}\right)^{\dagger} \Pi_{s_{1}, c_{1} \in\left[s_{1}, c_{1}\right]}>_{K i n}} \\
& =\frac{\sum_{[s, c]} \sum_{v \in V(\gamma(s, c \in[s, c]))} \frac{1}{2} \lim _{\epsilon, \epsilon^{\prime} \rightarrow 0}}{\times \Pi_{\left[s_{2}, c_{2}\right]}\left[\hat{H}_{v, \gamma(s, c)}^{\epsilon} \Pi_{s, c \in[s, c]}\right] \sum_{s, c \in[s, c]}<\Pi_{s, c} \mid\left(\hat{H}_{v}^{\epsilon^{\prime}}\right)^{\dagger} \Pi_{s_{1}, c_{1} \in\left[s_{1}, c_{1}\right]}>_{K i n},}
\end{aligned}
$$

where $D i f f_{\gamma}$ is the set of diffeomorphisms leaving the colored graph $\gamma$ invariant, $G S_{\gamma}$ denotes the graph symmetry quotient group Diff $f_{\gamma} /$ TDiff $_{\gamma}$ where TDiff $_{\gamma}$ is the diffeomorphisms which is trivial on the graph $\gamma$, and $n_{\gamma}$ is the number of elements in $G S_{\gamma}$. Note that we have used the resolution of identity trick in the fourth step. Since only finite number of terms in the sum over spin-scalar-networks $(s, c)$, cells $C \in$ $\mathcal{P}$, and diffeomorphism transformations $\varphi$ are non-zero respectively, we can interchange the sums and the limit. In the fifth step, we take the limit $C \rightarrow v$ and split the sum $\sum_{s, c}$ into $\sum_{[s, c]} \sum_{s, c \in[s, c]}$, where $[s, c]$ denotes the diffeomorphism equivalent class associated with $(s, c)$. Here we also use the fact that, given $\gamma(s, c)$ and $\gamma\left(s^{\prime}, c^{\prime}\right)$ which are different up to a diffeomorphism transformation, there is always a diffeomorphism $\varphi$ transforming the graph associated with $\hat{H}_{v, \gamma(s, c)}^{\epsilon} \Pi_{s, c}(v \in \gamma(s, c))$ to that of $\hat{H}_{v^{\prime} \gamma\left(s^{\prime}, c^{\prime}\right)}^{\epsilon} \Pi_{s^{\prime}, c^{\prime}}\left(v^{\prime} \in \gamma\left(s^{\prime}, c^{\prime}\right)\right)$ with $\varphi(v)=v^{\prime}$, hence $\Pi_{\left[s_{2}, c_{2}\right]}\left[\hat{H}_{v, \gamma(s, c)}^{\epsilon} \Pi_{s, c \in[s, c]}\right]$ is constant for different $(s, c) \in[s, c]$.

Since the term $\sum_{s, c \in[s, c]}<\Pi_{s, c} \mid\left(\hat{H}_{v}^{\epsilon^{\prime}}\right)^{\dagger} \Pi_{s_{1}, c_{1} \in\left[s_{1}, c_{1}\right]}>_{K i n}$ is independent of the parameter $\epsilon^{\prime}$, one can see that by fixing an arbitrary state-dependent triangulation $T\left(\epsilon^{\prime}\right)$,

$$
\begin{aligned}
& \sum_{s, c \in[s, c]}<\Pi_{s, c}\left(\hat{H}_{v}^{\epsilon^{\prime}}\right)^{\dagger} \Pi_{s_{1}, c_{1} \in\left[s_{1}, c_{1}\right]}>_{K i n} \\
= & \sum_{\varphi}<U_{\varphi} \Pi_{s, c}\left(\hat{H}_{v}^{\epsilon^{\prime}}\right)^{\dagger} \Pi_{s_{1}, c_{1} \in\left[s_{1}, c_{1}\right]}>_{K i n} \\
= & \sum_{\varphi}<\hat{H}_{v, \varphi(\gamma(s, c))}^{\epsilon^{\prime}} U_{\varphi} \Pi_{s, c}\left[\Pi_{s_{1}, c_{1} \in\left[s_{1}, c_{1}\right]}>_{K i n}\right. \\
= & \sum_{\varphi}<U_{\varphi} \hat{H}_{\varphi^{-1}\left(\epsilon^{\prime}\right), \gamma(s, c)}^{\varphi^{-1}\left(\epsilon^{\prime}\right)} \Pi_{s, c} \mid \Pi_{s_{1}, c_{1} \in\left[s_{1}, c_{1}\right]}>_{K i n} \\
= & \frac{\Pi_{\left[s_{1}, c_{1}\right]}\left[\hat{H}_{v \in V(\gamma(s, c)), \gamma(s, c)}^{\varphi^{-1}\left(\epsilon^{\prime}\right)} \Pi_{s, c}\right]}{}
\end{aligned}
$$

where $\varphi$ are the diffeomorphism transformations spanning the diffeomorphism equivalent class $[s, c]$. Note that the kinematical inner product in above sum is non-vanishing if and only if $\varphi(\gamma(s, c)))$ coincides with the extended graph obtained from certain skeleton $\gamma\left(s_{1}, c_{1}\right)$ by the action of $\left(\hat{H}_{v}^{\epsilon^{\prime}}\right)^{\dagger}$ and $v \in V(\varphi(\gamma(s, c)))$, i.e., the scale $\varphi^{-1}\left(\epsilon^{\prime}\right)$ of the diffeomorphism images of the tetrahedrons added by the action coincides with the scale of certain tetrahedrons in $\gamma(s, c)$ and $\varphi^{-1}(v)$ is a vertex in $\gamma(s, c)$. Then we can express 
the matrix elements (46) as:

$$
\begin{aligned}
& <\Pi_{\left[s_{1}, c_{1}\right]}|\hat{\mathbf{M}}| \Pi_{\left[s_{2}, c_{2}\right]}>_{\text {Diff }} \\
= & \sum_{[s, c]} \sum_{v \in V(\gamma(s, c \in[s, c]))} \frac{1}{2} \lim _{\epsilon, \epsilon^{\prime} \rightarrow 0} \overline{\Pi_{\left[s_{2}, c_{2}\right]}\left[\hat{H}_{v, \gamma(s, c)}^{\epsilon} \Pi_{s, c \in[s, c]}\right]} \Pi_{\left[s_{1}, c_{1}\right]}\left[\hat{H}_{v, \gamma(s, c)}^{\epsilon^{\prime}} \Pi_{s, c \in[s, c]}\right] \\
= & \sum_{[s, c]} \sum_{v \in V(\gamma(s, c \in[s, c]))} \frac{1}{2} \frac{\left.\hat{H}_{v}^{\prime} \Pi_{\left[s_{2}, c_{2}\right]}\right)\left[\Pi_{s, c \in[s, c]}\right]}{\left(\hat{H}_{v}^{\prime} \Pi_{\left[s_{1}, c_{1}\right]}\right)\left[\Pi_{s, c \in[s, c]}\right] .}
\end{aligned}
$$

From Eq. (48) and the result that the master constraint operator $\hat{\mathbf{M}}$ is densely defined on $\mathcal{H}_{\text {Diff }}$, it is obvious that $\hat{\mathbf{M}}$ is a positive and symmetric operator on $\mathcal{H}_{\text {Diff }}$. Hence, it is associated with a unique self-adjoint operator $\hat{\mathbf{M}}$, called the Friedrichs extension of $\hat{\mathbf{M}}$. We relabel $\hat{\mathbf{M}}$ to be $\hat{\mathbf{M}}$ for simplicity. In conclusion, there exists a positive and self-adjoint operator $\hat{\mathbf{M}}$ on $\mathcal{H}_{\text {Diff }}$ corresponding to the master constraint (35). It is then possible to obtain the physical Hilbert space of the coupled system by the direct integral decomposition of $\mathcal{H}_{\text {Diff } f}$ with respect to $\hat{\mathbf{M}}$.

Note that the quantum constraint algebra can be easily checked to be anomaly free. Eq.(44) assures that the master constraint operator commutes with finite diffeomorphism transformations, i.e.,

$$
\left[\hat{\mathbf{M}}, \hat{U}_{\varphi}^{\prime}\right]=0 .
$$

Also it is obvious that the master constraint operator commutes with itself,

$$
[\hat{\mathbf{M}}, \hat{\mathbf{M}}]=0 .
$$

So the quantum constraint algebra is precisely consistent with the classical constraint algebra 36 in this sense. As a result, the difficulty of the original Hamiltonian constraint algebra can be avoided by introducing the master constraint algebra, due to the Lie algebra structure of the latter.

\section{Acknowledgments}

The authors would like to acknowledge the referee for helpful criticism on the original manuscript. This work is supported in part by NSFC (10205002). Muxin Han would also like to acknowledge the support from the fellowship and the assistantship of LSU, Hearne Foundation of LSU, and funding from Advanced Research and Development Activity.

\section{References}

[1] T. Thiemann, Modern Canonical Quantum General Relativity, (Cambridge University Press, in press), (Draft preprint: $\mathrm{gr}-\mathrm{qc} / 0110034)$.

[2] C. Rovelli, Quantum Gravity, (Cambridge University Press, 2004). 
[3] T. Thiemann, Lectures on loop quantum gravity, (Preprint: gr-qc/0210094).

[4] A. Ashtekar and J. Lewandowski, Background independent quantum gravity: A status report, Class. Quantum Grav. 21, R53 (2004), (preprint: gr-qc/0404018).

[5] M. Han, W. Huang, and Y. Ma, Fundamental structure of loop quantum gravity, (preprint: gr-qc/0509064).

[6] A. Ashtekar, New variables for classical and quantum gravity, Phys. Rev. Lett. 57, 2244 (1986).

[7] J. Barbero, Real Ashtekar variables for Lorentzian signature spacetimes, Phys. Rev. D 51, 5507 (1995).

[8] A. Ashtekar, J. Lewandowski, D. Marolf, J. Mourão, and T. Thiemann, Quantization of diffeomorphism invariant theories of connections with local degrees of freedom, J. Math. Phys. 36, 6456 (1995).

[9] T. Thiemann, A length operator for canonical quantum gravity, J. Math. Phys. 39, $3372(1998)$.

[10] C. Rovelli and L. Smolin, Discreteness of area and volume in quantum gravity, Nucl. Phys. B 442, 593, (1995).

[11] A. Ashtekar and J. Lewandowski, Quantum theory of geometry I: Area operators, Class. Quantum Grav. 14, A55 (1997).

[12] A. Ashtekar and J. Lewandowski, Quantum theory of geometry II: Volume operators, Adv. Theor. Math. Phys. 1, 388 (1997).

[13] Y. Ma and Y. Ling, $\hat{Q}$ operator for canonical quantum gravity, Phys. Rev. D 62, 104021 (2000).

[14] T. Thiemann, Quantum spin dynamics (QSD), Class. Quantum Grav. 15, 839 (1998).

[15] T. Thiemann, Quantum spin dynamics (QSD): II. The kernel of the Wheeler DeWitt constraint operator, Class. Quantum Grav. 15, 875 (1998).

[16] T. Thiemann, The phoenix project: Master constraint programme for loop quantum gravity, (preprint: gr-qc/0305080).

[17] T. Thiemann, Quantum spin dynamics (QSD): VII. The master constraint, (preprint: gr-qc/0510011).

[18] M. Han and Y. Ma, Master constraint operator in loop quantum gravity, (Preprint: gr-qc/0510014).

[19] A. Ashtekar, J. Lewandowski, and H. Sahlmann, Polymer and Fock representations for a scalar field, Class. Quantum Grav. 20, L11 (2003). 
[20] W. Kaminski, J. Lewandowski and M. Bobienski, Background independent quantizations: the scalar field I, (preprint: gr-qc/0508091).

[21] T. Thiemann, Kinematical Hilbert spaces for fermionic and Higgs quantum field theories, Class. Quantum Grav. 15, 1487 (1998).

[22] T. Thiemann, Quantum spin dynamics (QSD): V. Quantum gravity as the natural regulator of the Hamiltonian constraint of matter quantum field theories, Class. Quantum Grav. 15, 1281 (1998).

[23] M. Han, Y. Ma, Y. Ding, and L. Qin, Hamiltonian analysis of $n$-dimensional Palatini gravity with matter, Mod. Phys. Lett. A20 725 (2005), (preprint: gr-qc/0503024).

[24] C. Rovelli and L. Smolin, Spin networks and quantum gravity, Phys. Rev. D 52, 5743, (1995).

[25] H. Sahlmann and T. Thiemann, Toward the QFT on curved spacetime limit of QGR I: A general scheme, Class. Quantum Grav. 23, 867 (2006), (preprint: gr-qc/0207030).

[26] M. Bojowald, Loop quantum cosmology, Living Rev. Relativity, 8, (2005), 11.

[27] M. Han, The solutions of Wheeler-DeWitt equation of loop quantum gravity with matter, (in preparation)

[28] M. Reed and B. Simon, Methods of Modern Mathematical Physics II: Fourer Analysis, Self-adjointness, (Academic Press, 1975), Page 177.

[29] R. M. Wald, Quantum Field Theory in Curved Spacetime and Black Hole Thermodynamics, (The University of Chicago Press, 1994).

[30] E. E. Flanagan and R. M. Wald, Does backreaction enforce the everaged null energy condition in semiclassical gravity, Phys. Rev. D54, 6233 (1996).

[31] K.V. Kuchar, Covariant factor ordering of gauge systems, Phys. Rev. D34, 3044 (1986).

P. Hajicek and K.V. Kuchar, Constraint quantization of parametrized relativistic gauge system in curved spacetimes, Phys. Rev. D41, 1091 (1990). 